\title{
Welche Unternehmen berufen Vorstandsvorsitzende und andere Vorstände als externe Kontrolleure? Eine empirische Analyse der Präsenz von externen Vorständen in den Aufsichtsräten deutscher Grossunternehmen
}

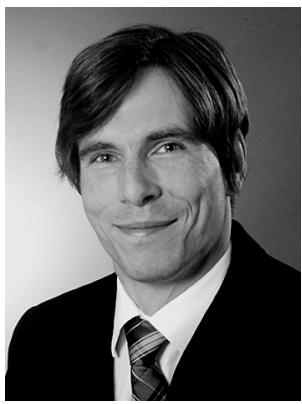

den Beitrag werden erstmals für deutsche Grossunternehmen systematisch unternehmensspezifische Faktoren für die Präsenz externer Vorstände in den Aufsichtsräten und zeitliche Entwicklungstendenzen anhand eines aktuellen Datensatzes für die Jahre 1997 bis 2008 empirisch analysiert. Unter anderem nimmt die Präsenz externer Vorstände im Aufsichtsrat mit der Unternehmensgrösse und dem Stimmrechtsanteil der Unternehmen aus dem untersuchten Datensatz zu. Die getrennte Auswertung unterschiedlicher Gruppen von externen Vorständen im Aufsichtsrat zeigt, dass externe Vorstandsvorsitzende im Vergleich zu anderen externen Vorstandsmitgliedern signifikant häufiger Mandate in erfolgreicheren, stärker diversifizierten sowie gelisteten Unternehmen ausüben. Die Kontrolle für den Einfluss der Einführung der Reform des Steuerrechts sowie der Veröffentlichung des Deutschen Corporate Governance Kodex im Jahr 2002 führt zudem zu qualitativ vergleichbaren Ergebnissen. Die Ergebnisse verdeutlichen die weiterhin hohe Bedeutung von Mehrfachmandaten als Instrument der Corporate Governance in Unternehmen, die nicht schwerpunktmässig dem Kredit- oder Versicherungsgewerbe zuzuordnen sind.

The appointment of external managers to the supervisory board is common practice between large companies. During the past decades, German companies were linked by a dense network of personal linkages via executive and supervisory boards. Based on panel data for the period from 1997 to 2008 the paper empirically analyses trends and firm-specific factors that explain the presence of outside executives on the supervisory boards of large German companies. The results of the econometric estimations reveal a positive relation between company size and stock ownership of the companies in the sample and the presence of outside executives on the supervisory board. Separate estimations for the 
group of chairmen and regular executives on the executive committee show that chairmen are more likely to join the supervisory boards of more successful, diversified and listed companies. Finally, the analysis controls for the effect of the coefficients during the period before and the period following the tax reform and the adoption of the German Corporate Governance Code in 2002 what yielded similar results and points to the ongoing importance of multiple directorships for corporate governance in large German corporations.

\section{Einleitung}

Die Analyse der Zusammensetzung von Aufsichtsräten und in diesem Zusammenhang insbesondere die Rolle von Mehrfachmandatsträgern ist seit vielen Jahren Gegenstand einer steigenden Zahl wissenschaftlicher Forschungsarbeiten. Auch in der politischen und öffentlichen Diskussion werden unterschiedliche Aspekte der Corporate Governance regelmässig aufgegriffen. Als prominente Beispiele lassen sich die in jüngster Zeit verstärkt diskutierte Frage nach der Sicherstellung einer angemessenen Höhe von Vorstandsvergütungen und die Forderung nach personeller Vielfalt bei der Zusammensetzung von Aufsichtsratsgremien zur Gewährleistung einer effektiven Kontrolle anführen. ${ }^{1}$

Die vorliegende Arbeit adressiert im Unterschied zu vorausgegangenen Studien zu deutschen Unternehmen auf dem Gebiet der empirischen Corporate Governance-Forschung nicht den Zusammenhang zwischen Mehrfachmandatsträgern und spezifischen Erfolgsgrössen wie dem Unternehmenserfolg (vgl. z. B. Andres/Lehmann 2010; Balsmeier et al. 2010) oder der Vorstandsvergütung (vgl. u. a. Balsmeier/Peters 2009 sowie Entorf et al. 2009), sondern konzentriert sich auf die Nachfrage nach externen Führungskräften als Mitglieder des Kontrollgremiums. Während Balsmeier/Buchwald (2011) in einer früheren Studie Bestimmungsfaktoren für die Entsendung deutscher Vorstandsvorsitzender in externe Kontrollgremien auswerten, erfolgt die empirische Analyse dieser Untersuchung aus der Perspektive der berufenden Unternehmen. Den Schwerpunkt der Untersuchung bildet entsprechend die Identifikation und Analyse von unternehmensspezifischen Charakteristika, anhand derer die Präsenz von externen Vorständen und Vorstandsvorsitzenden in den Aufsichtsräten deutscher Grossunternehmen über einen langen Zeitraum von 12 Jahren zwischen 1997 und 2008 erklärt werden kann.

In Übereinstimmung mit einer aktuellen empirischen Untersuchung von Fablenbrach et al. (2010a), die für eine Stichprobe von über 4.800 US-amerikanischen Unternehmen Bestimmungsfaktoren für die Berufung externer CEOs herausarbeiten, zeigt sich, dass externe Vorstände signifikant häufiger in grössere Unternehmen berufen werden. Fablenbrach et al. (2010a) heben in ihrer Analyse die besondere Bedeutung von CEOs als externe Boardmitglieder hervor, da diese aufgrund ihrer Erfahrung und Reputation zum einen die Kontroll- und Beratungsqualität im Board erhöhen und damit zum Erfolg des Unternehmens beitragen. Zum anderen kann das blosse Engagement eines CEO als Zertifizierung für das empfangende Unternehmen interpretiert werden, selbst wenn der tatsächliche Einfluss eines CEO im Board zu vernachlässigen ist. Für den zweiten Erklärungsansatz spricht der Befund der Autoren, dass externe CEOs während ihrer Amtsdauer keine spürbaren

1 Siehe hierzu z. B. die aktuellen Empfehlungen des Deutschen Corporate Governance Kodex in der Fassung vom 26. Mai 2010. Für einen umfassenden Überblick zu der Vergütungsproblematik siehe die Studie von Noll et al. (2011). 
Auswirkungen auf das empfangende Unternehmen erkennen lassen. Um der möglicherweise herausgehobenen Stellung eines Vorstandsvorsitzenden im deutschen Corporate Governance-System Rechnung zu tragen, werden in der vorliegenden Untersuchung separate Schätzungen für externe Vorstandsvorsitzende als Mitglieder des Aufsichtsrates und gewöhnliche Vorstandsmitglieder durchgeführt. Es zeigt sich, dass die Präsenz beider Gruppen in grösseren Unternehmen höher ist und mit der Zahl amtierender Vorstandsmitglieder und deren externen Kontrollmandaten abnimmt. Allerdings findet sich ein signifikant positiver Zusammenhang zwischen dem Unternehmenserfolg und der Diversifizierung des Unternehmens sowie einer Börsennotierung ausschliesslich für die Gruppe externer Vorstandsvorsitzender im Aufsichtsrat.

Die deutsche Unternehmenslandschaft war über viele Jahre geprägt durch ein enges Netzwerk aus wechselseitigen Kapitalverflechtungen und Beziehungen über Mehrfachmandatsträger (vgl. Beyer 1996 sowie Höpner/Krempel 2004). Institutionelle Veränderungen in Deutschland wie die Reform des Steuerrechts im Jahr 2002, die für Unternehmen den Verkauf von Unternehmensbeteiligungen steuerfrei stellte, die Veröffentlichung des Deutschen Corporate Governance Kodex im gleichen Jahr, oder die zunehmende Fokussierung der Banken auf das Investmentbankinggeschäft begünstigten die fortschreitende Auflösung gegenseitiger Kapitalbeteiligungen, zu einem wesentlichen Teil getrieben durch den Rückzug der grossen deutschen Finanzdienstleistungsinstitute (Heinze 2004; Monopolkommission 2006; Dittmann et al. 2010). Gleichzeitig zeigt die deskriptive Analyse der Unternehmen in dem vorliegenden Beitrag, dass sich die Auflösung personeller Unternehmensverbindungen über Mehrfachmandatsträger in Industrie-, Handels- und Dienstleistungsunternehmen nur in einem vergleichbar geringen Masse vollzog (vgl. hierzu auch Rank 2006; Monopolkommission 2010). Um für einen möglichen Strukturbruch zu kontrollieren, wird im Rahmen der empirischen Untersuchung in weiteren Schätzungen der Einfluss der Koeffizienten auf die Wahrscheinlichkeit der Berufung externer Vorstände in den Aufsichtsrat in den Zeiträumen vor und nach Inkrafttreten der Reform des Steuerrechts und der Veröffentlichung des Corporate Governance Kodex im Jahr 2002 untersucht. Die Ergebnisse zeigen, dass die Unterschiede im Erklärungsgehalt und der Signifikanz der Koeffizienten in beiden Zeiträumen als tendenziell gering einzuschätzen sind.

Im folgenden zweiten Abschnitt werden zunächst theoretische Ansätze zur Erklärung von Unternehmensverflechtungen über Mehrfachmandatsträger diskutiert und empirische Befunde skizziert. Auf dieser Grundlage werden im Anschluss Hypothesen abgeleitet. Für die empirische Überprüfung der Hypothesen wird in Abschnitt 3 die Datenbasis beschrieben. In Abschnitt 4 wird die Wahl der ökonometrischen Methoden diskutiert und die Ergebnisse der empirischen Untersuchung zusammengefasst. Eine abschliessende Würdigung der Resultate und der Verweis auf weiterführende aktuelle Forschungsfragen erfolgt in Abschnitt 5 .

\section{Literaturüberblick und Ableitung der Hypothesen}

\subsection{Theoretische Grundlagen}

Eine steigende Zahl umfassender empirischer Forschungsarbeiten zu US-amerikanischen Unternehmen befasst sich mit relevanten Aspekten und Wirkungen der bestehenden Corporate Governance-Strukturen (für einen Überblick vgl. unter anderem Shleifer/Vishny 1997; Hermalin 2005; Bebchuk et al. 2009 sowie Adams et al. 2010). Eine herausragende 
Bedeutung wird in der akademischen Diskussion dem empirischen Phänomen personeller Unternehmensverflechtungen über Mehrfachmandatsträger beigemessen. Das deutsche Corporate Governance-System weist gegenüber angelsächsisch geprägten Ländern eine Reihe von institutionellen Unterschieden auf, insbesondere die personelle Trennung des geschäftsführenden und des Kontrollgremiums sowie gesetzliche Regelungen zur Mitbestimmung von Arbeitnehmervertretern (für eine umfassende Diskussion des institutionellen Rahmens in Deutschland und den USA siehe z. B. Beetz 2005; Fauver/Fuerst 2006 sowie Balsmeier/Buchwald 2011). Als wesentliche Aufgaben des Aufsichtsrats nennt der Deutsche Corporate Governance Kodex die regelmässige Beratung und Überwachung des Vorstands sowie die Beteiligung an Entscheidungen von grundlegender Bedeutung. ${ }^{2}$ Ungeachtet der unterschiedlichen institutionellen Rahmenbedingungen der verschiedenen Governance-Regime ist das Grundproblem der Sicherstellung einer effektiven Überwachung auf die Trennung von Eigentum und Kontrolle zurückführen und kann entsprechend durch eine klassische Prinzipal-Agenten-Beziehung abgebildet werden (Jensen/ Meckling 1976; Fama/Jensen 1983). Zur Beschreibung der Motive für personelle Unternehmensverflechtungen werden in der Literatur unterschiedliche Argumente aufgeführt. Nach Schreyögg/Papenheim-Tockhorn (1995) können personelle Verflechtungen als ein Instrument zum gezielten Aufbau einer Unternehmensverbindung eingesetzt werden. Aus dieser institutionellen Perspektive dienen entsprechende Verflechtungsbeziehungen unter anderem dem Ziel, Unsicherheiten auf den Beschaffungs- und Absatzmärkten zu reduzieren (Pfeffer 1972, 1992) oder eine effiziente Koordination von Transaktionen entlang der Wertschöpfungskette sicherzustellen (vgl. z. B. Pfannschmidt 1995 oder Balsmeier et al. 2010 für einen Überblick).

Bereits Schönwitz/Weber (1980) argumentieren aus einer betriebswirtschaftlichen Perspektive, dass eine Vielzahl parallel ausgeübter Ämter sowohl zu einer zeitlichen Überlastung führen als auch weitere Interessenkonflikte nach sich ziehen können, die möglicherweise den wirtschaftlichen Interessen verschiedener Stakeholdergruppen entgegenstehen. Neuere empirische Befunde deuten tendenziell auf eine abgeschwächte Kontrollintensität durch personelle Unternehmensverflechtungen hin (vgl. u. a. Fich/Shivdasani (2006) und Barnea/Guedj (2009) für ein Sample US-amerikanischer bzw. Andres/Lehmann (2010) für ein Sample deutscher Unternehmen). Entorf et al. (2009) sowie Balsmeier/Peters (2009) weisen einen positiven Zusammenhang zwischen der Zahl externer Kontrollmandate der Vorstandsmitglieder in deutschen Unternehmen und ihrer Bezüge nach. Allerdings sinkt die Vorstandsvergütung mit der Zahl externer Vorstände im Aufsichtsrat (siehe Entorf et al. 2009). Balsmeier et al. (2010b) zeigen, dass personelle Unternehmensverflechtungen mit einer signifikant höheren Fluktuation der Vorstandsmitglieder einhergehen und werten den Befund als Hinweis auf die Wirksamkeit der deutschen Corporate Governance. Auch Schönwitz/Weber (1980) erkennen positive Effekte von Mehrfachmandaten an, da Managementqualifikation eine knappe Ressource darstellt und externe Mandate demnach zu einer effizienten Allokation führen können, die die Management- und Kontrollqualität in den entsprechenden Gremien erhöht. Nach Biehler/Ortmann (1985) schränken bei der Rekrutierung von Kapitalvertretern die hohen und spezifischen Anforderungen an ein Aufsichtsratsmandat den Kreis der in Frage kommenden Personen relativ stark ein. Famal

2 Vgl. hierzu die Empfehlungen des Deutschen Corporate Governance Kodex in der Fassung vom 26. Mai 2010, Ziffer 5.1.1, und Ringleb et al. 2010. 
Jensen (1983) betonen, dass die Topmanager selbst Mehrfachmandate nutzen, um ihre besonderen Fähigkeiten und ihre Managementexpertise auf dem Arbeitsmarkt zu signalisieren. Mehrfachmandate sind für einen Manager daher mit einem höheren Bekanntheitsgrad verbunden und schaffen zusätzliche Geschäftskontakte (Mace 1986). Zudem können sich Topmanager über externe Kontrollmandate durch zusätzliche Unternehmens- und Brancheneinblicke weiter qualifizieren (Johnson/Apps 1979; Conyon/Read 2006). Vorstände erfolgreicher Unternehmen halten demnach eine höhere Zahl externer Mandate (Kaplan 1994). Conyon/Read (2006) zeigen allerdings auch, dass die für einen Manager optimale Zahl externer Mandate über der des entsendenden Unternehmens liegt. Ungeachtet der bestehenden Anreize, eine superoptimale Zahl externer Mandate auf sich zu vereinen, sind die Opportunitätskosten jedes zusätzlichen Mandats infolge von Zeitrestriktionen für einen Vorstand hoch. Die Bereitschaft eines Vorstands zur Ausübung externer Mandate hängt dabei von der spezifischen Situation im berufenden Unternehmen als auch von der ausgeübten Position und den Rahmenbedingungen im entsendenden Unternehmen ab. Booth/Deli (1996) zeigen, dass Vorstände in Unternehmen mit grösseren Wachstumsperspektiven seltener externe Mandate ausüben. Auch ist anzunehmen, dass die Opportunitätskosten eines Vorsitzenden des Vorstandsgremiums diejenigen eines gewöhnlichen Vorstandsmitglieds übersteigen. Fablenbrach et al. (2010a) nehmen eine Unterscheidung zwischen dem Angebot an externer Beratungs- und Kontrollexpertise durch Topmanager und der Nachfrage nach externer Expertise durch das berufende Unternehmen vor. Die Autoren argumentieren, dass CEOs ein mögliches Engagement in einem externen Board nach verschiedenen Kriterien evaluieren. Letztlich akzeptiert ein CEO ein Angebot, wenn das Gesamtpaket einen ausreichenden Nutzenzugewinn erwarten lässt. Neben der Vergütung stellen auch die Arbeitsbelastung bzw. die Opportunitätskosten insgesamt sowie nicht-monetäre Vergünstigungen wichtige Kriterien für die Entscheidung dar (Linck et al. 2009). Erwarten Manager, dass das zu kontrollierende Unternehmen in eine finanzielle Schieflage gerät, tendieren sie zur Wahrung ihrer Reputation dazu, das entsprechende externe Mandat niederzulegen (siehe Fablenbrach et al. 2010b).

Betreffend die Nachfrage nach externen Vorständen lassen sich zwei wesentliche Motive festhalten. Neben der Sicherstellung unabhängiger externer Kontrolle wird in der Literatur auch auf die Beratungsfunktion externer Manager verwiesen (Fich 2005; Adams/Ferreira 2007). Die Berufung externer Vorstände sollte sich daher wie zuvor skizziert vorteilhaft auf die Corporate Governance und den wirtschaftlichen Erfolg eines Unternehmens auswirken. Ein besonderes Mass sowohl an Autorität als auch an Erfahrung wird hierbei externen CEOs zugesprochen. Eine Reihe von empirischen Studien befasst sich zudem mit der Bedeutung des beruflichen Hintergrundes von Mehrfachmandatsträgern hinsichtlich ihrer Beratungsfunktion. ${ }^{3}$ Fablenbrach et al. (2010a) argumentieren zudem, dass die Berufung einer bekannten Führungspersönlichkeit in das Board eine wertvolle Zertifizierung des Unternehmens zur Folge haben kann. Dieser eher psychologische Effekt wird damit begründet, dass die Gewinnung eines auf seine Reputation bedachten Managers als Boardmitglied eine positive Signalwirkung für die verschiedenen Stakeholdergruppen entfaltet, selbst wenn der tatsächliche Einfluss auf die Geschäftstätigkeit gering ist. Auch diesem Ef-

3 Vgl. z. B. Güner et al. (2008) und Dittmann et al. (2010) für Bankenvertreter bzw. Agrawal/Knoeber (2001) und Miwa/Ramseyer (2005) für externe Boardmitglieder mit einem politischen Hintergrund. 
fekt ist mit zunehmendem Bekanntheitsgrad der berufenen Person ein stärkeres Gewicht beizumessen.

\subsection{Hypothesen}

Die Ausführungen in den vorangegangenen Abschnitten haben gezeigt, dass die Präsenz externer Vorstände im Aufsichtsrat das Ergebnis von Angebot an und Nachfrage nach externer Managementexpertise darstellt. Die vorliegende Arbeit stellt hierbei die Berufung externer Führungskräfte in den Vordergrund. Von einer simultanen Analyse der Angebotsseite muss infolge der begrenzten Datenlage hinsichtlich spezifischer Variablen zur Messung der Anreizstrukturen bzw. der Opportunitätskosten externer Vorstände wie z. B. die Vergütung des Kontrollmandats, die erwartete Anzahl der Sitzungen des Aufsichtsrats bzw. die Mitwirkung in Ausschüssen abgesehen werden. In diesem Abschnitt werden Bestimmungsfaktoren und ihr erwarteter Einfluss auf die Präsenz externer Vorstände im Aufsichtsrat diskutiert. Die Ableitung der Hypothesen stützt sich in Anlehnung an bestehende empirische Studien auf die zuvor skizzierten theoretischen Ansätze, insbesondere die $\mathrm{Hu}-$ mankapital-, Transaktionskosten- und Ressourcen-Abhängigkeits-Theorie sowie die Prinzipal-Agenten-Theorie, wobei auch bestehende empirische Befunde zur Abschätzung der Richtung des Zusammenhangs berücksichtigt werden. Hierbei erfolgt die Diskussion sowohl aus der Perspektive des berufenden Unternehmens wobei auch die anzunehmenden Anreizstrukturen potentieller Mandatsträger, die den Interessen der Anteilseigner möglicherweise entgegenstehen, diskutiert werden.

In Übereinstimmung mit der zitierten Literatur ist Aufsichtsratsmitgliedern, die ein paralleles Amt als Vorstandsvorsitzender ausüben, aufgrund ihrer besonderen Stellung ein hervorgehobenes Gewicht beizumessen. Allerdings sollte sich die Richtung des zu erwartenden Zusammenhangs zwischen den zu skizzierenden Determinanten und der Präsenz externer Vorstandsvorsitzender sowie gewöhnlichen Vorstandsmitgliedern nicht unterscheiden. Aus diesem Grund wird bei der Formulierung der Hypothesen keine Unterscheidung zwischen beiden Gruppen vorgenommen.

\section{Unternehmenserfolg}

Ein unterdurchschnittlicher Unternehmenserfolg kann neben anderen Faktoren auf eine unzureichende Qualifikation des amtierenden Managements zurückgeführt und ein Bedarf nach externem Humankapital abgeleitet werden (Zimmermann 2010). Die gleiche Argumentation lässt sich auch auf die Beratungs- und Kontrollexpertise des Aufsichtsrates übertragen. Die Berufung externer Vorstände kann eine unabhängige Analyse der Situation begünstigen. Die stärkere Unabhängigkeit externer Vorstände gegenüber dem Management kann zudem die Durchsetzung organisatorischer und personeller Veränderungen und eine strategische Neuausrichtung vereinfachen (Weisbach 1988). Diesem erhöhten Bedarf an externen Beratern in schwierigen Zeiten stehen geringe Anreize potentieller Mandatsträger gegenüber, ein entsprechendes Mandat zu akzeptieren. Eine auf seine Reputation bedachte Führungskraft wird diese durch ein riskantes und zeitintensives Engagement in einem unterdurchschnittlich erfolgreichen Unternehmen nur ungern gefährden. Zudem sind die Einflussmöglichkeiten eines Aufsichtsrates gegenüber einem möglicherweise neu berufenen Vorstandsvorsitzenden, der sich als starker Reformer profilieren kann, begrenzt. Zudem sprechen die Befunde von Fablenbrach et al. (2010b) dafür, dass externe Vorstän- 
de das Unternehmen gerade dann verlassen, wenn die erwartete Rendite in den Folgeperioden sinkt. Dieses Ergebnis lässt einen positiven Zusammenhang zwischen dem Unternehmenserfolg und der Zahl externer Vorstände im Aufsichtsrat erwarten. Zudem lässt sich vor dem Hintergrund des Reputationsargumentes unterstellen, dass erfolgreiche Unternehmen in besonderem Masse potentielle externe Mandatsträger anziehen.

\section{H1: „Zwischen der Zahl externer Vorstände im Aufsichtsrat und dem Unternehmenser- folg besteht ein positiver Zusammenhang. “}

\section{Komplexität des Unternehmens}

Grössere Unternehmen weisen tendenziell eine höhere Zahl an Geschäftsbeziehungen zu Unternehmen verschiedener Branchen im In- und Ausland auf (vgl. Booth/Deli 1996). Wie im vorausgegangenen Abschnitt aufgezeigt wurde, können Unternehmensverflechtungen über Mehrfachmandatsträger sowohl aus transaktionskostentheoretischen Überlegungen als auch aus dem Blickwinkel der Ressourcen-Abhängigkeits-Theorie einen effizienten Mechanismus darstellen, um Transaktionen zu Zulieferunternehmen und Kunden zu vereinfachen und Unsicherheiten abzubauen. Entsprechend sind externe Vorstände mit spezifischen Kenntnissen und unterschiedlichen Branchenerfahrungen im Kontrollgremium geeignet, sowohl einen Beitrag zu leisten, die komplexen Strukturen in grösseren Unternehmen zu verstehen, als auch Geschäftskontakte zu anderen Unternehmen herzustellen bzw. zu festigen. Für den unterstellten positiven Zusammenhang spricht auch der Befund von Beyer (1996), der in einer empirischen Untersuchung zu deutschen Unternehmen einen positiven Zusammenhang zwischen der Unternehmensgrösse und dem Grad personeller Unternehmensverflechtungen für das Jahr 1992 feststellt. Balsmeier/Buchwald (2011) zeigen, dass Vorstandsvorsitzende in grösseren Unternehmen häufiger externe Kontrollmandate ausüben. Zudem bietet die Tätigkeit in grösseren Unternehmen einem potentiellen Mandatsträger eine Reihe monetärer und nicht-monetärer Vergünstigungen. Aufsichtsratsmandate in grösseren Unternehmen sind mit einem höheren Grad an Verantwortung und öffentlicher Aufmerksamkeit und damit Prestige verbunden und eröffnen den Mandatsträgern weitreichende Geschäftskontakte (Fablenbrach et al. 2010a). Linck et al. (2009) stellen zudem eine höhere Vergütung in grösseren Unternehmen fest.

Fablenbrach et al. (2010a) finden neben der Grösse des Unternehmens auch einen positiven Zusammenhang zwischen dem Alter des Unternehmens und der Zahl externer Mandatsträger im Board. Es lässt sich unterstellen, dass Unternehmen, die sich über viele Jahre auf dem Markt etabliert haben, durch eine relativ hohe Zahl von Geschäfts- und persönlichen Beziehungen der Mandatsträger zu anderen Unternehmen gekennzeichnet sind. Der Befund lässt sich auch als Indiz dafür werten, dass Vorstände externe Kontrollmandate in älteren Unternehmen bevorzugen. Zum einen ist das Risiko eines Engagements in einem etablierten Unternehmen als geringer einzuschätzen. Mandate in einem älteren, angesehenen und in der Regel bekannten Unternehmen sind zum anderen als prestigeträchtiger und damit attraktiver für einen potentiellen Mandatsträger einzustufen. Boone et al. (2007) bilden die Komplexität eines Unternehmens mit der Grösse, dem Alter sowie der Zahl der Geschäftsfelder ab und finden wie auch Coles et al. (2008) und Linck et al. (2008) einen positiven Zusammenhang zwischen der Komplexität und der Zahl externer Manager im Board. 
Als zusätzliches Mass für die Komplexität eines Unternehmens wird entsprechend der Diversifizierungsgrad, gemessen an der Zahl der Wirtschaftszweige, in denen das Unternehmen wesentliche Umsätze erzielt, herangezogen. Analog der Argumentation für die Wirkung der Unternehmensgrösse steigt die Zahl externer Zuliefer- und Abnehmerkontakte mit der Komplexität des Unternehmens. Zugleich gewinnt das Argument der Branchenexpertise mit einer zunehmenden Zahl von Geschäftsfeldern an Bedeutung. Sowohl aus einer transaktionskostentheoretischen Perspektive als auch der Ressourcen-AbhängigkeitsTheorie folgend kann unterstellt werden, dass mit zunehmender Komplexität die Zahl der Zuliefer- und Abnehmerbeziehungen zu Unternehmen entlang der Wertschöpfungskette zunehmen und personelle Verflechtungen entsprechend einen wirksamen Mechanismus zur Koordination dieser Austauschbeziehungen zwischen Unternehmen darstellen.

H2: „Die Zahl externer Vorstände im Aufsichtsrat nimmt mit steigender Komplexität eines Unternehmens zu."

\section{Variablen zur Struktur des Vorstands- und Aufsichtsratsgremiums}

Zusätzlich werden Variablen zur Struktur von Vorstand und Aufsichtsrat in die empirische Untersuchung einbezogen. US-amerikanische Autoren argumentieren, dass mit der Grösse des Board of Directors der Koordinationsaufwand steigt bzw. die Kontrollintensität abnimmt (siehe u. a. Faleye 2003). Yermack (1996) findet einen negativen Zusammenhang zwischen der Grösse des Board und dem Unternehmenserfolg. Coles et al. (2008) zeigen in einer differenzierten Analyse, dass komplexe Unternehmen einen erhöhten Beratungsbedarf aufweisen. In komplexen Unternehmen zeigt sich ein positiver Zusammenhang zwischen dem Unternehmenserfolg und der Grösse des Board bzw. der Zahl externer Boardmitglieder. Dieser Befund spricht für die Notwendigkeit einer umfassenderen $\mathrm{Hu}-$ mankapitalausstattung in komplexen Unternehmen, die sich auch durch den Zukauf externer Managementexpertise sicherstellen lässt. Unterstellt man, dass die Grösse des Vorstandsgremiums in der zweistufigen deutschen Struktur zu einem gewissen Grad die Komplexität eines Unternehmens widerspiegelt, lässt sich ein möglicher positiver Zusammenhang zwischen der Präsenz externer Vorstände im Aufsichtsrat und der Zahl der amtierenden Vorstandsmitglieder ableiten. Hinsichtlich des Aufsichtsratsgremiums ist festzuhalten, dass in deutschen Unternehmen die Mindestzahl der Mitglieder des Aufsichtsrates an die Grösse des Unternehmens infolge gesetzlicher Bestimmungen geknüpft ist ( $\int 7$ Abs. 1 MitbestG). Die Grösse des Aufsichtsrates wird daher zu einem gewissen Grad durch die Grösse des Unternehmens bestimmt. Unter bestimmten Voraussetzungen sind Unternehmen im Sample vom Geltungsbereich des Mitbestimmungsgesetzes ausgenommen (siehe hierzu die Erläuterungen von Höpner/Müllenborn 2010). Der zuvor erwartete positive Zusammenhang zwischen Unternehmensgrösse und externen Vorständen sollte sich daher aufgrund der gesetzlichen Vorschriften auch für die Grösse des Aufsichtsrates einstellen.

H3.1: „Mit zunehmender Grösse des Aufsichtsratsgremiums steigt die Zahl externer Vorstände im Aufsichtsrat."

Ein möglicherweise negativer Einfluss der Zahl der amtierenden Vorstandsmitglieder auf die Berufung externer Vorstände in den Aufsichtsrat kann sich daraus ergeben, dass trotz höherer Komplexität kein weiterer Bedarf an externer Managementexpertise besteht, da das Vorstandsgremium bereits über ausreichendes Humankapital verfügt. Um die mög- 
liche Nachfrage nach externer Beratung näher zu beleuchten, wird als weitere Kontrollvariable die Zahl der externen Kontrollmandate des eigenen Vorstandes in die Schätzungen einbezogen. Mit zusätzlichen Kontakten zu weiteren Unternehmen können Vorstände ihren eigenen Erfahrungshorizont gemäss der Argumentation von Conyon/Read (2006) erweitern und auf diesem Wege Beratungsexpertise bzw. individuelles Humankapital über externe Mehrfachmandatsträger im Aufsichtsrat substituieren.

H3.2: „Die Häufigkeit der Berufung externer Vorstände in Aufsichtsrat nimmt mit zunehmender der Grösse des Vorstandsgremiums bzw. der Anzahl externer Kontrollmandate des Vorstands ab."

\section{Variablen zur Kapitalstruktur}

Die Struktur und Konzentration der Eigentümer wie auch die Notierung an der Börse sind als wichtige Erklärungsfaktoren für die Präsenz externer Vorstände im Aufsichtsrat zu interpretieren (für eine umfassende Diskussion des Zusammenhangs zwischen Eigentümerstruktur und Corporate Governance siehe auch Becht et al. 2002). Gerade für grosse börsennotierte Unternehmen ist der Zertifizierungshypothese eine besondere Bedeutung beizumessen. Die Berufung einer bekannten, qualifizierten und angesehenen Führungskraft, insbesondere eines Vorsitzenden des Vorstandes einer anderen grossen Gesellschaft, sendet ein wichtiges Signal an den Finanzmarkt und unterstreicht den Anspruch einer soliden Führung und Kontrolle des Unternehmens.

Für den Mandatsträger selbst erfordert die Tätigkeit als Aufsichtsrat in einem grossen gelisteten Unternehmen aufgrund der umfassenden gesetzlichen Bestimmungen einen hohen Zeitaufwand und fundierte fachliche Kenntnisse zur ordnungsgemässen Erfüllung des Amtes. Gleichzeitig bedeutet die Ausübung eines Aufsichtsratsmandats in einem grossen gelisteten Unternehmen und hierbei besonders in einem der DAX-30-Konzerne ein hohes Mass an Prestige und öffentlicher Wahrnehmung. Ein entsprechendes Kontrollmandat eröffnet einem Vorstand neben dem Zugewinn an Reputation auch künftige Karriereoptionen, die seine Opportunitätskosten möglicherweise aufwiegen.

H4.1: „Unternehmen, die an einer deutschen Wertpapierbörse gelistet sind, berufen häufiger externe Vorstände in den Aufsichtsrat."

Mit zunehmender Höhe des Streubesitzes sinkt die Wahrscheinlichkeit der Präsenz eines oder mehrerer grosser Anteilseigner. Hieraus können für die Governance des Unternehmens negative Wirkungen entstehen. Grosse Blockholder, wie beispielsweise Privatpersonen, institutionelle Investoren oder andere Unternehmen, entsenden üblicherweise Vertreter in den Aufsichtsrat des Beteiligungsunternehmens, um die Unternehmenspolitik gemäss den eigenen Interessen und Zielsetzungen zu beeinflussen (Shleifer/Vishny 1997). Die Abwesenheit eines bedeutenden Anteilseigners kann wiederum die Kontrollintensität vermindern und dem aktiven Vorstand Verhaltensspielräume eröffnen. Informationsasymmetrien begünstigen in Anlehnung an die Prinzipal-Agenten-Theorie die Durchsetzung von opportunistischem Verhalten. Die Auswirkungen einer entsprechenden Konstellation auf die Wahrscheinlichkeit der Berufung externer Vorstände sind aus einer theoretischen Perspektive nicht eindeutig zu bestimmen. Es lässt sich argumentieren, dass externe Vorstände im Aufsichtsrat infolge ihrer Unabhängigkeit gegenüber dem Vorstand und zur Wahrung ihrer Reputation die Kontrollintensität erhöhen. Verfügen die amtierenden Mitglieder des 
Vorstandes über eine relativ ausgeprägte Machtstellung, sollten Anreize bestehen, die Berufung externer Kontrolleure in den Aufsichtsrat zu verhindern. Andere Autoren finden dagegen Hinweise, dass gerade Mehrfachmandatsträger eine niedrigere Kontrollintensität aufweisen (Fich/Shivdasani 2006). Zwar ist eine direkte Einflussnahme des Vorstandes auf die Berufung im Unterschied zu US-amerikanischen Unternehmen nicht möglich (zu der Beteiligung des CEO an der Auswahl neuer Boardmitglieder in US-amerikanischen Unternehmen siehe Shivdasani/Yermack 1999), da die Vertreter der Arbeitgeberseite gemäss $\$ 101$ Abs. 1 AktG durch die Hauptversammlung gewählt werden. Es ist allerdings anzunehmen, dass insbesondere der Vorsitzende des Vorstands Einfluss auf die zur Wahl vorzuschlagenden Kandidaten nehmen kann. Dies gilt umso mehr, wenn der Aufsichtsrat infolge einer unterjährigen Erweiterung oder des Ausscheidens eines Mitglieds die zur Beschlussfähigkeit erforderliche Mitgliederzahl nicht aufweist. $\$ 104$ Abs. 1 AktG sieht für diesen Fall die Bestellung eines Aufsichtsratsmitglieds durch das Gericht auf Vorschlag des Vorstands vor. Insgesamt sollte es dem Vorstand umso leichter fallen, einen favorisierten Kandidaten durchzusetzen, je stärker die Stimmrechtsanteile zersplittert sind. Im Unterschied zu der Annahme einer stärkeren Kontrolle durch externe Mandatsträger geht die Theorie sozialer Netzwerke davon aus, dass Mitglieder der obersten Entscheidungsgremien in Grossunternehmen einem begrenzten und abgeschotteten Kreis von Personen angehören, die durch langjährige gemeinsame soziale Kontakte gekennzeichnet sind und weitestgehend losgelöst von einer wirksamen Kontrolle durch die Eigentümer agieren können (vgl. u. a. Mizruchi 1996 und Windolf 1997). Trifft diese Annahme für deutsche Unternehmen zu, sollte mit zunehmendem Streubesitz die Einflussnahme der Anteilseigner auf die Unternehmenspolitik weiter abnehmen und die Präsenz externer Vorstände im Aufsichtsrat steigen.

\section{H4.2: „Der Anteil des Streubesitzes geht mit einer zunehmenden Zabl externer Vorstände im Aufsichtsrat einher."}

Ein positiver Einfluss auf die Zahl externer Vorstände lässt sich ebenfalls mit der Höhe des Stimmrechtsanteils von Unternehmen aus dem untersuchten Datensatz erwarten. Wie zuvor beschrieben, ist es gängige Praxis, dass grössere Kapitalgeber aus transaktionskostentheoretischen Überlegungen Personen ihres Vertrauens in den Aufsichtsrat des Beteiligungsunternehmens entsenden. In der deutschen Unternehmenslandschaft galt dies über lange Zeit in besonderem Masse für die grossen Unternehmen der Finanzdienstleistungsbranche, die erhebliche Kapitalbeteiligungen an Industrieunternehmen hielten und Mitglieder ihrer Vorstandsgremien in die Aufsichtsräte der Beteiligungsunternehmen entsandten (Monopolkommission 2006). Dittmann et al. (2010) stellen allerdings eine zunehmende Disparität zwischen gehaltenen Kapitalanteilen und der Präsenz von Bankenvertretern in Aufsichtsräten fest. Insgesamt ist ein positiver Zusammenhang zwischen der Höhe des Kapitalanteils von Unternehmen aus dem Datensatz und der Zahl externer Vorstände im Aufsichtsrat zu erwarten, die bestehende Beteiligungen überwachen.

H4.3: „Je grösser die Höhe der Stimmrechtsanteile, die von Unternehmen aus dem Untersuchungskreis gehalten werden, desto grösser ist die Zabl externer Vorstände im Aufsichtsrat." 


\section{Datenbasis und deskriptive Befunde}

Für die empirische Analyse der Bestimmungsfaktoren für die Präsenz externer Vorstände im Aufsichtsrat wurden Informationen aus verschiedenen Quellen genutzt. Die Definition der Variablen und die zugehörigen Quellen sind in Tabelle 1 zusammengefasst.

\begin{tabular}{ll}
\hline Variable & Beschreibung \\
\hline Verflechtungsvariablen: & \\
\hline $\begin{array}{l}\text { Externe Vorstände im AR } \\
\text { Dummy externe Vorstände }\end{array}$ & $\begin{array}{l}\text { Zahl der externen Vorstände im Aufsichtsrat } \\
\text { Dummy-Variable, nimmt den Wert eins an, } \\
\text { wenn mindestens ein externer Vorstand im }\end{array}$ \\
$\begin{array}{l}\text { Externe Vorstände (exkl. } \\
\text { Vorsitz) im AR }\end{array}$ & $\begin{array}{l}\text { Zahl der externen Vorstände (exkl. Vorstands- } \\
\text { vorsitzende) im Aufsichtsrat }\end{array}$ \\
$\begin{array}{l}\text { Dummy externe Vorstände } \\
\text { (exkl. Vorsitz) im AR }\end{array}$ & $\begin{array}{l}\text { Dummy-Variable, nimmt den Wert eins an, } \\
\text { wenn mindestens ein externer Vorstand (exkl. } \\
\text { Vorsitz) im Aufsichtsrat gezählt wird }\end{array}$ \\
$\begin{array}{l}\text { Externe Vorstandsvorsitzen- } \\
\text { de im AR }\end{array}$ & $\begin{array}{l}\text { Zahl der externen Vorstandsvorsitzenden im } \\
\text { Aufsichtsrat }\end{array}$ \\
$\begin{array}{l}\text { Dummy externe Vorstands- } \\
\text { Dorsitzende im AR }\end{array}$ & $\begin{array}{l}\text { Dummy-Variable, nimmt den Wert eins an, } \\
\text { wenn mindestens ein externer Vorstandsvorsit- } \\
\text { zender im Aufsichtsrat gezählt wird }\end{array}$ \\
Externe Mandate Vorstand & $\begin{array}{l}\text { Zahl der externen Kontrollmandate der Mit- } \\
\text { glieder des Vorstands }\end{array}$ \\
Unternehmenserfolg: &
\end{tabular}

Return on Investment Return on Investment = Jahresüberschuss nach Steuern/Gesamtkapital

Quellen

jeweils:

Geschäftsberichte, Monopol-

kommission

Bureau van Dijk:

„AMADEUS“ Datenbank

Komplexität des Unternehmens:

Beschäftigte Anzahl der Mitarbeiter des Unternehmens

Bureau van Dijk:

„AMADEUS“ Datenbank

Alter des Unternehmens Alter des Unternehmens in Jahren

Bureau van Dijk:

„AMADEUS“ Datenbank

Diversifikation

Anzahl der Geschäftsfelder (Klassifikation der Wirtschaftszweige (WZ 2003))

Geschäftsberichte,

Hoppenstedt:

„Companies \& Sectors“,

„Konzernstrukturdatenbank”

Variablen zur Kapitalstruktur:

\begin{tabular}{ll}
\hline Listung & $\begin{array}{l}\text { Dummy-Variable }=1 \text {, wenn das Unternehmen } \\
\text { einer deutschen Wertpapierbörse gelistet ist }\end{array}$ \\
Streubesitz & Anteil Streubesitz (in Prozent)
\end{tabular}

Kapitalanteil Unternehmen Stimmrechtsanteil Unternehmen aus dem Sample (in Prozent)

Bureau van Dijk:

„AMADEUS“ Datenbank

Hoppenstedt:

„Companies \& Sectors“, „Konzernstrukturdatenbank”

Hoppenstedt:

„Companies \& Sectors“, „Konzernstrukturdatenbank”

Charakteristika von Vorstand und Aufsichtsrat:

\begin{tabular}{lll}
\hline Mitglieder Vorstand & Zahl der Mitglieder des Vorstandes & $\begin{array}{l}\text { Geschäftsberichte, Monopol- } \\
\text { kommission }\end{array}$ \\
Mitglieder AR & Zahl der Mitglieder des Aufsichtsratsgremi- & $\begin{array}{l}\text { Geschäftsberichte, Monopol- } \\
\text { kommission }\end{array}$ \\
\hline
\end{tabular}

Tabelle 1: Definition der Variablen und Datenquellen 
Die Auswahl der Unternehmen orientiert sich an den 100 grössten Unternehmen in Deutschland, gemessen an ihrem Beitrag zur Wertschöpfung aller Unternehmen. Die relevanten Unternehmen wurden den Gutachten der Monopolkommission entnommen, die im Rahmen ihrer Konzentrationsberichterstattung turnusgemäss alle zwei Jahre personelle Verflechtungen zwischen den Unternehmen sowie Anteilseignerinformationen und weitere Begleitmerkmale zu den Grossunternehmen ausweist. Die erfassten Unternehmen stellen die grössten Akteure auf dem deutschen Markt dar und sind traditionell über personelle und kapitalmässige Verflechtungen eng miteinander verwoben. Der Vorteil des Panels besteht darin, dass die Unternehmen nicht auf einen Aktienindex limitiert sind und wichtige Verflechtungsbeziehungen auch zwischen nicht börsengelisteten Unternehmen erfasst werden können (siehe Höpner/Müllenborn 2010). Unternehmen, die dem Konsolidierungskreis eines Konzerns mit Sitz im Ausland angehören, wurden aus dem Datensatz entfernt. Ebenso wurde der Datensatz um Banken und Versicherungskonzerne bereinigt, da entsprechende Gesellschaften durch eingeschränkt vergleichbare Erfolgsgrössen gekennzeichnet sind. Daten für die Zwischenjahre wurden auf Grundlage des Untersuchungskreises der Vorperiode ermittelt. In das bereinigte Panel fliessen 604 Unternehmensjahre von insgesamt 74 Unternehmen ein. Bilanzdaten sowie weitere Unternehmensangaben wurden unterschiedlichen Datenbanken der privaten Anbieter Bureau van Dijk und des HoppenstedtVerlages sowie den publizierten Geschäftsberichten der Unternehmen entnommen.

In Tabelle 2 sind die deskriptiven Statistiken zu den für die Untersuchung relevanten Variablen aufgelistet. Der Return on Investment als das Verhältnis von Periodengewinn und Gesamtkapitaleinsatz wird als Masszahl für den bilanziellen Erfolg des Unternehmens herangezogen. Gegenüber der Gesamtkapitalrendite wird im Falle des Return on Investment von einer Korrektur um die auf Fremdkapitalgeber entfallenden Zinsen abgesehen. In der empirischen Analyse wurde zudem für alternative bilanzielle Erfolgsgrössen wie die Eigenkapitalrendite oder die jeweiligen Abweichungen vom Branchendurchschnitt kontrolliert, für die sich vergleichbare Ergebnisse zeigten. Von einer Einbeziehung marktwertbasierter Performancemasse wurde abgesehen, da nicht auf Marktreaktionen oder die Performance als zu erklärende Grösse abgestellt wird. Aus diesem Grunde wurde der Berücksichtigung nicht börsennotierter Unternehmen in der Untersuchung Vorrang vor der Verwendung marktwertbasierter Performancemasse als zusätzliche Kontrollgrösse eingeräumt.

Die Zahl der Beschäftigten als Proxy für die Unternehmensgrösse beläuft sich im Mittel auf 87.074 Personen bei einer Standardabweichung von 104.888 Beschäftigten. Das durchschnittliche Alter der Unternehmen im Sample beträgt 81,9 Jahre. Die Zahl der Segmente als Mass für die Diversifizierung schwankt mit einem Mittelwert von 2,8 zwischen einem und neun Geschäftsfeldern. 60,1 Prozent der Unternehmen werden an einer Börse gehandelt. Die Kapitalstruktur der Unternehmen wird durch die Höhe des Streubesitzes sowie die Kapitalanteile, die kumuliert durch die Unternehmen im Sample gehalten werden, abgebildet. Durchschnittlich liegen über alle Unternehmen und Jahre 31,7 Prozent des Kapitals in Streubesitz. 11,5 Prozent der Anteile entfallen auf andere Unternehmen im Sample. Die Entwicklung beider Kategorien über die Zeit spiegelt die im ersten Abschnitt beschriebene Auflösung des Netzwerkes aus wechselseitigen Kapitalbeteiligungen zwischen deutschen Grossunternehmen wider. Sank der Anteil des Streubesitzes im Untersuchungszeitraum 1997 bis 2008 moderat von 36,8 auf 29,2 Prozent, reduzierte sich im gleichen Zeitraum der auf Grossunternehmen entfallende Kapitalanteil um 54,6 Prozent von 16,3 auf 7,4 Prozent. Im Mittel setzen sich das Vorstands- und Aufsichtsratsgremium eines Un- 
ternehmens aus 6,4 bzw. 17,0 Mitgliedern zusammen. Tabelle 2 weist ferner die deskriptiven Statistiken zu den personellen Verflechtungsvariablen der Untersuchung aus.

\begin{tabular}{|c|c|c|c|c|c|}
\hline & Beobachtungen & $\begin{array}{l}\text { Mittel- } \\
\text { wert }\end{array}$ & $\begin{array}{l}\text { Std. } \\
\text { Abw. }\end{array}$ & Min & $\operatorname{Max}$ \\
\hline \multirow{3}{*}{$\begin{array}{l}\text { Externe Vorstände im AR } \\
\text { Dummy externe Vorstände im AR } \\
\text { Externe Vorstände (exkl. Vorsitz) } \\
\text { im AR }\end{array}$} & 604 & 0,917 & 1,321 & 0 & 8 \\
\hline & 604 & 0,518 & - & 0 & 1 \\
\hline & 604 & 0,389 & 1,054 & 0 & 7 \\
\hline $\begin{array}{l}\text { Dummy externe Vorstände (exkl. } \\
\text { Vorsitz) im AR }\end{array}$ & 604 & 0,207 & - & 0 & 1 \\
\hline $\begin{array}{l}\text { Externe Vorstandsvorsitzende im } \\
\text { AR }\end{array}$ & 604 & 0,528 & 0,727 & 0 & 3 \\
\hline $\begin{array}{l}\text { Dummy externe Vorstandsvorsit- } \\
\text { zende im AR }\end{array}$ & 604 & 0,407 & - & 0 & 1 \\
\hline Externe Mandate Vorstand & 604 & 0,977 & 1,670 & 0 & 9 \\
\hline Return on Investment & 604 & 0,040 & 0,063 & $-0,193$ & 0,775 \\
\hline Beschäftigte & 604 & 87.074 & 104.888 & 4.571 & 520.334 \\
\hline Alter des Unternehmens & 604 & 81,882 & 53,843 & 0 & 249 \\
\hline Diversifikation & 604 & 2,795 & 1,860 & 1 & 9 \\
\hline Listung & 604 & 0,609 & - & 0 & 1 \\
\hline Streubesitz & 604 & 31,695 & 33,321 & 0 & 99,2 \\
\hline Kapitalanteil Unternehmen & $\begin{array}{l}604 \\
604\end{array}$ & $\begin{array}{r}11,542 \\
6,366\end{array}$ & $\begin{array}{r}21,641 \\
3,404\end{array}$ & $\begin{array}{l}0 \\
1\end{array}$ & $\begin{array}{r}100,0 \\
26\end{array}$ \\
\hline Mitglieder AR & 604 & 17,033 & 4,307 & 3 & 24 \\
\hline
\end{tabular}

Anmerkungen: Der Modalwert stellt den gängigen Lageparameter für nominalskalierte Merkmale dar. Im Falle der in der Tabelle ausgewiesenen Dummyvariablen lassen sich die Angaben zum Mittelwert als Verteilung der Variablen interpretieren. Der Modalwert lässt sich hierbei aus der Verteilung ableiten.

Tabelle 2: Deskriptive Statistiken

Ergänzend stellt Abbildung 1 den zeitlichen Verlauf der aus dem Kreis der 100 grössten Unternehmen empfangenen externen Vorstände im Aufsichtsrat dar. Deren kumulierte Zahl beträgt insgesamt 0,92 Mandate. Hiervon werden durchschnittlich rund 0,39 Mandate von regulären Vorständen und 0,53 Mandate von externen Vorstandsvorsitzenden ausgeübt. Alle drei Verflechtungsvariablen weisen im Zeitraum 1997 bis 2008 nur relativ geringe Änderungen auf. Als zusätzliche Kontrollvariable ist die Zahl der externen Kontrollmandate der Mitglieder des eigenen Vorstands in Tabelle 2 aufgelistet. Im Durchschnitt üben 0,98 Mitglieder des Vorstandes externe Kontrollmandate aus. 

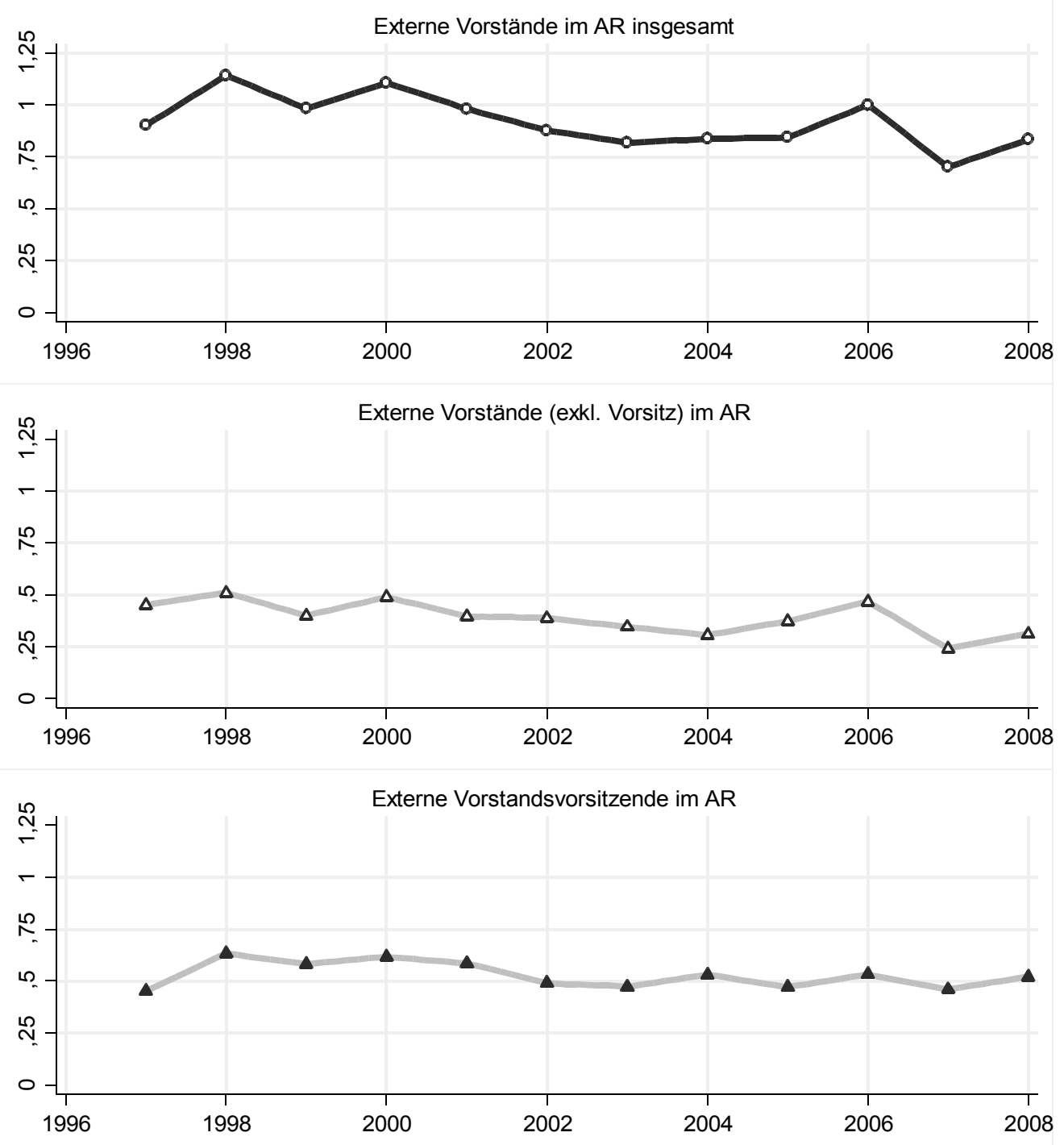

Abbildung 1: Entwicklung der durchschnittlichen Anzahl von externen Vorständen in den Aufsichtsratsgremien

In Tabelle 3 sind die Korrelationskoeffizienten aller für die empirische Untersuchung herangezogenen Variablen dargestellt. 


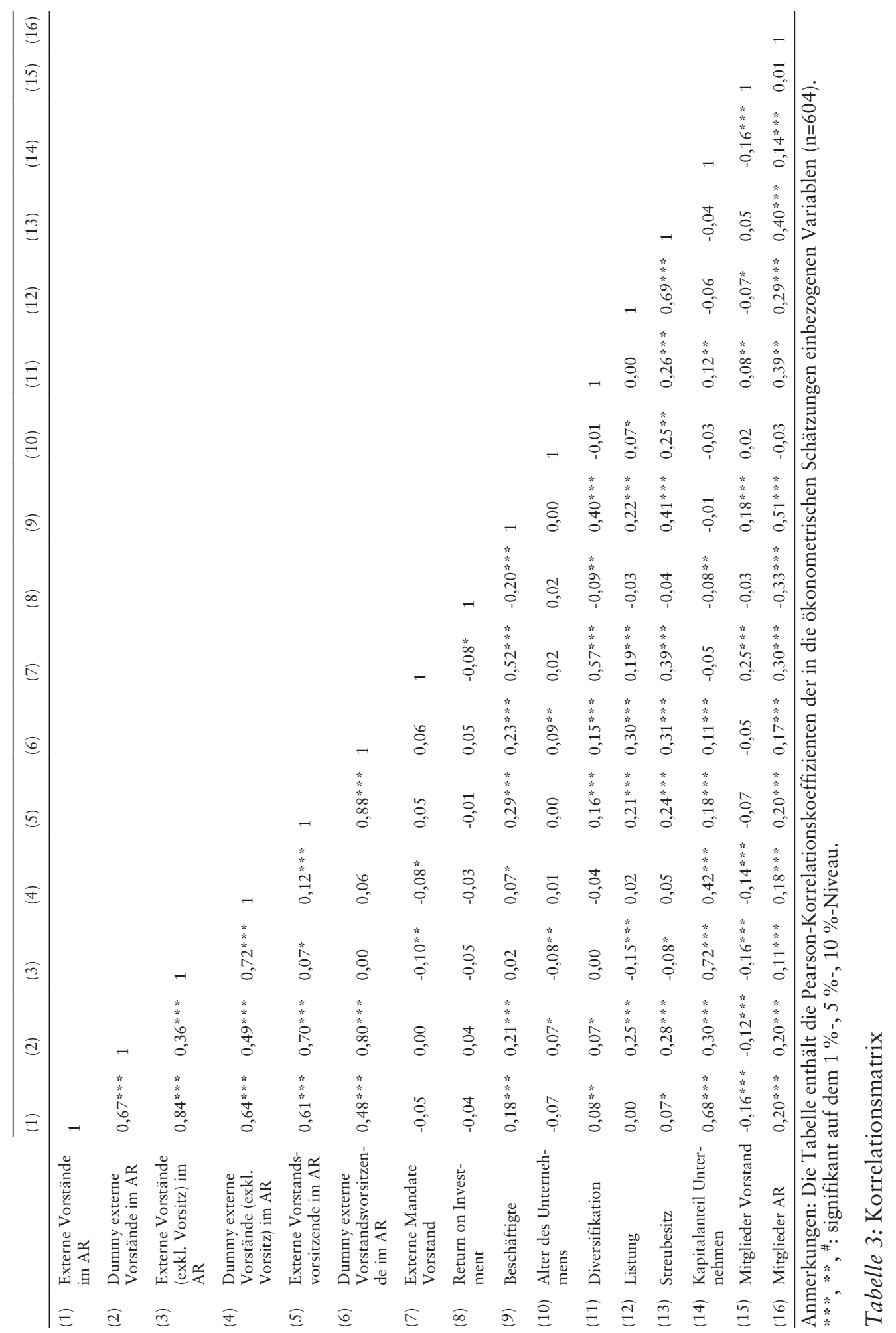


Tabelle 4 fasst die Ergebnisse des Vergleichs der Mittelwerte zwischen der Gruppe der Unternehmen mit mindestens einem externen Vorstand bzw. Vorstandsvorsitzenden und der Gruppe der Unternehmen ohne externe Vorstände im Aufsichtsrat zusammen. Unternehmen, die externe Vorstände in ihren Aufsichtsrat berufen haben, sind tendenziell grösser, weisen einen höheren Streubesitz sowie einen höheren Stimmrechtsanteil von Unternehmen aus dem untersuchten Datensatz auf und haben eine signifikant höhere Zahl an Aufsichtsratsmitgliedern. Gleichzeitig ist das Vorstandsorgan mit Ausnahme des Subsamples für externe Vorstandsvorsitzende signifikant kleiner. Weiterhin zeigen die Ergebnisse, dass Vorstandsvorsitzende häufiger in stärker diversifizierten und gelisteten Unternehmen als externe Kontrolleure tätig sind.

\begin{tabular}{cccc}
\hline \multirow{2}{*}{ Variable } & $A R$ & $A R$ & Mean Comparison \\
& obne externe $\ldots$ & mit externen ... & t-Test $(t$-Wert) \\
\hline
\end{tabular}

(a) ... Vorständeln insgesamt

Return on Investment

Beschäftigte

Alter des Unternehmens

Diversifikation

$$
0,038
$$

$0,042 \quad-0,893$

$76.339,930$

77,924

2,660

0,485

$7.053,830$

$-2,426 *$

Listung

21,936

85,562

$-1,748^{\#}$

Streubesitz

4,896

2,920

$-1,729^{\#}$

Kapitalanteil Unternehmen

6,797

$0,725-6,215 * *$

Mitglieder Vorstand

16,127

40,767

$-7,254 * *$

Mitglieder AR

0,973

$17,721-7,818 * * *$

Externe Mandate Vorstand

5,965

$2,956 * *$

Beobachtungen

291

$17,875-5,049 * *$

$0,981 \quad-0,0607$

\begin{tabular}{lrrr}
\hline & \multicolumn{3}{c}{} \\
\cline { 2 - 3 } & \multicolumn{3}{c}{ (b) ... Vorstände/n (exkl. Vorsitzende) } \\
\cline { 2 - 4 } Return on Investment & 0,041 & 0,036 & 1,017 \\
Beschäftigte & $84.592,370$ & $96.584,200$ & $-1,137$ \\
Alter des Unternehmens & 81,633 & 82,840 & $-0,204$ \\
Diversifikation & 2,833 & 2,648 & 0,999 \\
Listung & 0,605 & 0,624 & $-0,380$ \\
Streubesitz & 30,865 & 34,872 & $-1,220$ \\
Kapitalanteil Unternehmen & 6,886 & 29,383 & $-6,853 * * *$ \\
Mitglieder Vorstand & 6,612 & 5,424 & $5,187 * * *$ \\
Mitglieder AR & 16,637 & 18,552 & $-5,797 * * *$ \\
\hline
\end{tabular}




\begin{tabular}{lrrr}
\hline Variable & $\begin{array}{c}\text { AR } \\
\text { ohne externe } \ldots\end{array}$ & $\begin{array}{c}\text { AR } \\
\text { mit externen } \ldots\end{array}$ & $\begin{array}{c}\text { Mean Comparison } \\
t \text {-Test }(t \text {-Wert })\end{array}$ \\
\hline Externe Mandate Vorstand & 1,044 & 0,720 & $2,597 * * *$ \\
\hline Beobachtungen & 479 & \multicolumn{2}{c}{125} \\
\hline
\end{tabular}

(c) ... Vorstandsvorsitzende/n

Return on Investment

Beschäftigte

Alter des Unternehmens

Diversifikation

Listung

Streubesitz

Kapitalanteil Unternehmen

Mitglieder Vorstand

Mitglieder AR

Externe Mandate Vorstand
0,037

$75.725,880$

77,885

2,564

0,489

23,199

9,544

6,511

16,441

0,894

Beobachtungen

358

$\begin{array}{rl}0,044 & -1,091 \\ 103.589,000 & -3,268 * * * \\ 87,699 & -2,190 * * \\ 3,130 & -3,597 * * * \\ 0,785 & -7,932 * * * \\ 44,057 & -7,871 * * * \\ 14,450 & -2,752 * * * \\ 6,154 & 1,404 \\ 17,894 & -4,217 * * * \\ 1,098 & -1,500\end{array}$

Anmerkungen: Die Tabelle enthält zur Aufdeckung struktureller Differenzen zwischen der Gruppe von Unternehmen mit und ohne (a) externen Vorständen insgesamt, (b) externen Vorständen (exkl. Vorstandsvorsitzenden) und (c) externen Vorstandsvorsitzenden im Aufsichtsrat die Ergebnisse des Two-Group Mean Comparison t-Tests (Spalte 4). ${ }^{* * *}, * *$, : signifikant auf dem $1 \%-, 5 \%-, 10 \%$-Niveau.

Tabelle 4: Mittelwertvergleich

\section{Empirische Methodik und Schätzergebnisse}

\subsection{Vorbemerkungen zur Methodik}

Bei der Auswahl geeigneter ökonometrischer Modelle ist die spezifische Struktur der Anzahl externer Vorstandsmandate zu berücksichtigen. Zum einen nehmen die Verflechtungsvariablen nur ganzzahlige nichtnegative Ausprägungen an. Abbildung 2 veranschaulicht zum anderen die stark linkszentrierte Verteilung der drei Verflechtungsvariablen. In 48,2 Prozent aller Fälle ist kein Vorstand eines weiteren Unternehmens im Aufsichtsgremium aktiv. Für Vorstandsvorsitzende ergibt sich ein entsprechender Anteil von 59,3 bzw. für die Gruppe der gewöhnlichen externen Vorstandsmitglieder ein Wert von 79,3 Prozent. Aus Abbildung 2 ist abzulesen, dass die Kurvenverläufe näherungsweise durch eine Poisson-Verteilung beschrieben werden können. Ein Goodness-of-Fit- $\chi^{2}$-Test für die Poisson-Verteilung lässt sich für keine der drei Verflechtungsvariablen ablehnen. 

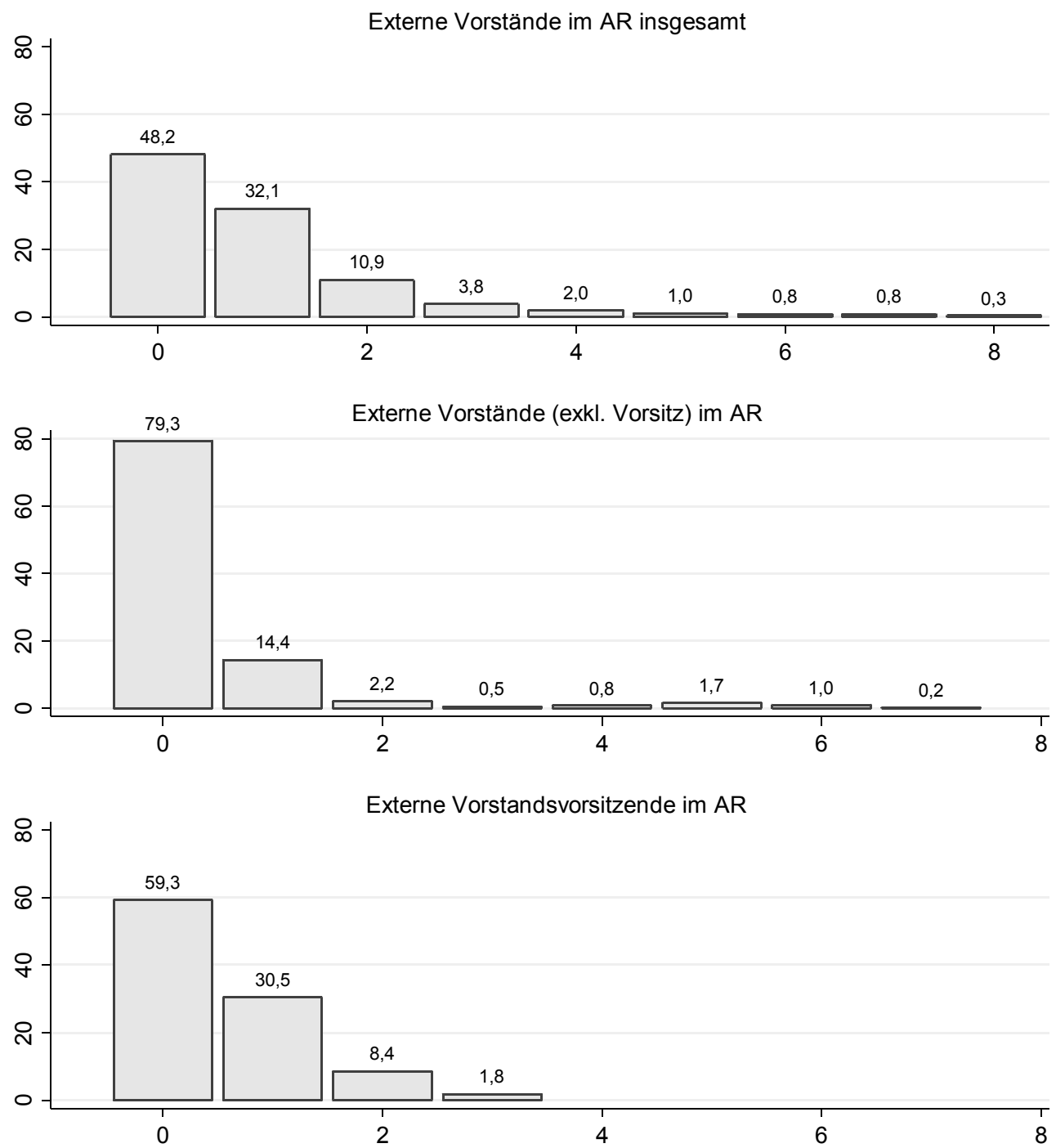

Abbildung 2: Häufigkeit von externen Vorstandsmitgliedern und Vorstandsvorsitzenden in den Aufsichtsratsgremien

Um der speziellen Struktur der abhängigen Variablen Rechnung zu tragen, werden für die ökonometrische Analyse Zähldatenmodelle angewendet. Im Unterschied zu StandardOLS-Regressionen berücksichtigen Zähldatenverfahren ausschliesslich nichtnegative und ganzzahlige Ausprägungen der abhängigen Variablen (für weiterführende Erläuterungen zu Count-Data Modellen siehe z. B. Cameron/Trivedi 1998 oder Winkelmann 2008). Im Folgenden werden entsprechend Poisson-Regressionen mit robusten Standardfehlern geschätzt, die konsistente Schätzwerte selbst im Falle einer Überdispersion der abhängigen 
Variable liefern. Entsprechende Tests zeigen hinsichtlich aller abhängigen Verflechtungsvariablen eine signifikante Überstreuung an. Alternativ wurden die Schätzungen auf der Grundlage von Negativ-Binomial-Modellen wiederholt, die eine Überdispersion der abhängigen Variable explizit berücksichtigen (Cameron/Trivedi 2010). Die Ergebnisse der Modellschätzungen führten zu nahezu identischen Ergebnissen hinsichtlich der Stärke und Signifikanz der erklärenden Variablen. Als ergänzender Test auf Robustheit werden in den folgenden beiden Abschnitten die Ergebnisse entsprechender Logit-Regressionen diskutiert. Die abhängigen Variablen nehmen entsprechend den Wert eins an, wenn mindestens ein externer Vorstand bzw. Vorstandsvorsitzender im Aufsichtsrat gezählt wird.

\subsection{Ergebnisse der Modellschätzungen}

Im folgenden Abschnitt werden die Ergebnisse der Poisson-Regressionen zur Erklärung der Präsenz externer Vorstände in den Aufsichtsratsgremien der Unternehmen im Panel vorgestellt. Tabelle 5 gibt in der Modellspezifikation (a) den Zusammenhang zwischen den erklärenden Variablen und der kumulierten Anzahl aller externen Vorstände im Aufsichtsrat an. In den Modellen (b) bzw. (c) wird der Einfluss der Koeffizienten auf die Zahl der gewöhnlichen Vorstandsmitglieder bzw. auf die Zahl der externen Vorstandsvorsitzenden im Aufsichtsrat analysiert. Alle Modelle wurden mit robusten Standardfehlern geschätzt und enthalten Perioden- und Industriedummies, um für Zeit- bzw. Industrieeffekte zu kontrollieren. Die erklärenden Variablen fliessen mit den um eine Periode verzögerten Werten in die Schätzungen ein, um die Endogenitätsproblematik abzumildern. ${ }^{4}$ In Tabelle 5 werden die marginalen Effekte der einzelnen Koeffizienten mit den zugehörigen z-Werten berichtet.

Die Modellschätzungen (a) und (c) zeigen einen signifikant positiven Zusammenhang zwischen dem Return on Investment der Vorperiode und der Zahl externer Vorstände im Aufsichtsrat. Für die Gruppe der gewöhnlichen Vorstandsmitglieder ergibt sich ebenfalls ein positiver, jedoch insignifikanter Zusammenhang. Alternative Erfolgsmasse wie die Eigenkapitalrendite sowie die entsprechenden Abweichungen vom Branchendurchschnitt führen zu identischen Ergebnissen. Der Befund stützt die im zweiten Abschnitt formulierte Hypothese 1, dass Vorstände häufiger Kontrollmandate in erfolgreichen Unternehmen ausüben. Dieser positive Zusammenhang gilt jedoch nur für Vorsitzende des Vorstands von Grossunternehmen. Es lässt sich annehmen, dass gerade Vorstandsvorsitzende durch ihren hohen Bekanntheitsgrad und ihre Unabhängigkeit stärker als externe Kontrolleure nachgefragt werden und auch selbst Anreize besitzen, Kontrollmandate in erfolgreichen Unternehmen zum Ausbau ihres Einflusses und ihrer Reputation anzunehmen. Alternative Schätzungen (nicht berichtet) liefern jedoch nur für die Gruppe von Vorstandsvorsitzenden schwache Hinweise für die These, dass diese seltener Kontrollmandate in Unternehmen ausüben, die in der Vorperiode einer sinkenden Rendite ausgesetzt waren.

4 Alle Schätzungen wurden mit nicht verzögerten Koeffizienten wiederholt, was zu vergleichbaren Ergebnissen führte. Alternativ wurden die Modelle in Anhang 3 und 4 mit den um zwei bzw. drei Perioden verzögerten erklärenden Variablen geschätzt, was ebenfalls zu vergleichbaren Ergebnissen führte. 


\begin{tabular}{|c|c|c|c|}
\hline & (a) & (b) & (c) \\
\hline & $\begin{array}{c}\text { Externe Vorstände } \\
\text { im AR }\end{array}$ & $\begin{array}{c}\text { Externe Vorstände } \\
\text { (exkl. Vorsitz) } \\
\text { im AR }\end{array}$ & $\begin{array}{l}\text { Externe Vorstands- } \\
\text { vorsitzende im AR }\end{array}$ \\
\hline \multirow[t]{2}{*}{ Return on Investment } & $1,738 * *$ & 0,275 & $1,067 * * *$ \\
\hline & $(4,88)$ & $(0,94)$ & $(4,50)$ \\
\hline \multirow[t]{2}{*}{ Log (Beschäftigte) } & $0,260 * * *$ & $0,043 * *$ & $0,204 * * *$ \\
\hline & $(7,15)$ & $(2,64)$ & $(6,68)$ \\
\hline \multirow[t]{2}{*}{ Alter des Unternehmens } & 0,001 & 0,000 & 0,001 \\
\hline & $(0,89)$ & $(0,78)$ & $(1,17)$ \\
\hline \multirow[t]{2}{*}{ Diversifikation } & $-0,037 * * *$ & $-0,027 * * *$ & $0,023 * *$ \\
\hline & $(-2,77)$ & $(-4,51)$ & $(2,02)$ \\
\hline \multirow[t]{2}{*}{ Listung } & 0,013 & $-0,059$ & $0,192 * * *$ \\
\hline & $(0,15)$ & $(-1,48)$ & $(3,04)$ \\
\hline \multirow[t]{2}{*}{ Streubesitz } & $0,004 * * *$ & $0,001^{\#}$ & 0,001 \\
\hline & $(2,98)$ & $(1,77)$ & $(1,25)$ \\
\hline \multirow[t]{2}{*}{ Kapitalanteil Unternehmen } & $0,015 * *$ & $0,005 * *$ & $0,004 * * *$ \\
\hline & $(16,56)$ & $(8,39)$ & $(4,86)$ \\
\hline \multirow[t]{2}{*}{ Mitglieder Vorstand } & $-0,063 * * *$ & $-0,025 * *$ & $-0,035 * * *$ \\
\hline & $(-5,39)$ & $(-4,02)$ & $(-3,60)$ \\
\hline \multirow[t]{2}{*}{ Mitglieder AR } & $0,030 * *$ & $0,023 * *$ & 0,001 \\
\hline & $(2,82)$ & $(4,96)$ & $(0,18)$ \\
\hline \multirow[t]{2}{*}{ Externe Mandate Vorstand } & $-0,076 * *$ & $-0,026 * *$ & $-0,055 * * *$ \\
\hline & $(-3,39)$ & $(-2,31)$ & $(-3,17)$ \\
\hline Beobachtungen & 604 & 604 & 604 \\
\hline Pseudo $\mathrm{R}^{2}$ & 0,263 & 0,439 & 0,107 \\
\hline Log Likelihood & $-627,909$ & $-326,785$ & $-515,624$ \\
\hline
\end{tabular}

Anmerkungen: Die Tabelle enthält Poisson-Regressionen zur Erklärung der Präsenz externer Vorstände im Aufsichtsrat. Als abhängige Variablen werden (a) die Zahl externer Vorstände im Aufsichtsrat insgesamt, (b) die Zahl externer Vorstände (exkl. Vorstandsvorsitzende) und (c) die Zahl externer Vorstandsvorsitzender im Aufsichtsrat herangezogen. Die exogenen Variablen fliessen mit den um eine Periode verzögerten Werten in die Schätzungen ein. In allen Schätzungen wurden Perioden- und Industriedummies verwendet. Berichtet werden die marginalen Effekte der einzelnen Koeffizienten mit den zugehörigen z-Werten in Klammern. Alle Modelle wurden mit robusten Standardfehlern geschätzt. ***,**\#: signifikant auf dem $1 \%-, 5 \%-, 10 \%$-Niveau.

Tabelle 5: Bestimmungsfaktoren für die Präsenz externer Vorstände im Aufsichtsrat 
Ein ebenfalls positiver und in allen Modellspezifikationen signifikanter Einfluss auf die Zahl externer Vorstände im Aufsichtsrat lässt sich gemäss Hypothese 2 für die Grösse des Unternehmens feststellen. Allerdings ist der Einfluss der Unternehmensgrösse auf die Zahl externer Vorstandsvorsitzender grösser als für die Gruppe der gewöhnlichen Vorstände. Alternativ wurde die logarithmierte Bilanzsumme als Proxy für die Grösse des Unternehmens überprüft, die zu vergleichbaren Ergebnissen führte.

Zwischen dem Alter des Unternehmens und der Verflechtung des Aufsichtsrates konnte kein signifikanter Zusammenhang identifiziert werden. Auch die Berücksichtigung eines quadratischen Terms in Anlehnung an Linck et al. (2008) zur Überprüfung eines nichtlinearen Zusammenhangs lieferte keine signifikanten Ergebnisse. Dagegen übt der Grad der Diversifikation einen signifikanten Einfluss auf die Zahl externer Vorstände im Aufsichtsrat aus. Betrachtet man wiederum die Gruppe der Vorstandsvorsitzenden und der gewöhnlichen empfangenen Vorstandsmitglieder, zeigen sich gegenläufige Effekte. Während mit jedem zusätzlichen Geschäftsfeld die Präsenz externer Vorsitzender im Aufsichtsrat um durchschnittlich 2,3 Prozent steigt, sinkt sie für gewöhnliche Vorstandsmitglieder um 2,7 Prozent. Insgesamt liefern die im Rahmen von Hypothese 2 erläuterten Variablen zur Abbildung der Komplexität eines Unternehmens ein gemischtes Bild. Während sich der Einfluss der Unternehmensgrösse für alle untersuchten Gruppen externer Kontrolleure als signifikant positiv herausstellt, gilt dies im Hinblick auf den Diversifizierungsgrad nur für externe Vorstandsvorsitzende. Als mögliche Erklärung für diese Befunde liesse sich argumentieren, dass Vorstandsvorsitzende aufgrund der Anforderungen an ihre Position im Vorstandsgremium über tiefergehende und breitere Fachkenntnisse sowie Managementexpertise verfügen als ein gewöhnlicher Vorstand, der in der Regel die Verantwortung für ein bestimmtes Ressort zu tragen hat. ${ }^{5}$ Diese Charakteristika machen ihn zu einem wichtigen Berater gerade in diversifizierten Grossunternehmen mit komplexeren Strukturen.

Die hervorgehobene Bedeutung von externen Vorstandsvorsitzenden als Mitgliedern des Aufsichtsrates zeigt sich auch hinsichtlich einer möglichen Börsennotierung des empfangenden Unternehmens. Die Wahrscheinlichkeit der Präsenz eines externen Vorstandsvorsitzenden ist signifikant höher in gelisteten Unternehmen. Für gewöhnliche Vorstandsmitglieder lässt sich ein insignifikant negativer Zusammenhang feststellen. Vorstandsvorsitzende scheinen Kontrollmandate in gelisteten Unternehmen zum Ausbau ihrer Reputation zu nutzen. Das Ergebnis unterstützt auch die These einer Zertifizierung des empfangenden Unternehmens, das mit der Berufung eines angesehenen und bekannten Vorstandsvorsitzenden eines anderen Grossunternehmens eine nachhaltige und erfolgreiche Geschäftspolitik anzeigt. Betrachtet man den Einfluss der Höhe des Streubesitzes (Hypothese 4.2) als weitere Kontrollvariable zur Kapitalstruktur, zeigen sich signifikante Ergebnisse nur für die externen Vorstände insgesamt und die Gruppe der Nicht-Vorsitzenden. Im Einklang mit Hypothese 4.3 besteht dagegen ein positiver und signifikanter Zusammenhang zwischen der Höhe des von Unternehmen aus dem untersuchten Datensatz gehaltenen Kapitalanteils und der Mitwirkung externer Vorstände im Aufsichtsrat. Das Ergebnis lässt sich als Hinweis werten, dass Unternehmen Mitglieder ihres Vorstands in Beteiligungsunter-

5 Unter anderem koordiniert der Vorsitzende die Arbeit des Vorstandsgremiums und fungiert als Ansprechpartner für den Aufsichtsratsvorsitzenden seines eigenen Unternehmens, mit dem er wesentliche operative und strategische Entwicklungen abstimmt. Vgl. hierzu z. B. die Empfehlungen des Deutschen Corporate Governance Kodex in der Fassung vom 26. Mai 2010, Ziffern 1 und 5.2. 
nehmen aus transaktionskostentheoretischen Überlegungen zur Kontrolle ihres Anteilsbesitzes entsenden.

Signifikant sind zudem die Koeffizienten zur Grösse der Vorstands- und Aufsichtsratsgremien in den empfangenden Unternehmen. Einerseits sinkt mit zunehmender Grösse des Vorstands die Häufigkeit der Berufung externer Vorstände in den Aufsichtsrat. Eine grössere Zahl amtierender spezialisierter Vorstände kann demnach möglicherweise den Bedarf nach externer Managementexpertise reduzieren. Andererseits besteht ein signifikant positiver Zusammenhang zwischen der Grösse des Aufsichtsratsgremiums und der Mitwirkung externer Vorstände im Gremium. Wie bei der Ableitung von Hypothese 3.2 in Abschnitt 2 diskutiert wurde, lässt sich die Grösse des Aufsichtsrates als Indikator für die Komplexität bzw. die Grösse eines Unternehmens interpretieren, womit sich der positive Zusammenhang begründen lässt. Einschränkend ist festzuhalten, dass die Grösse des Aufsichtsrates keinen signifikanten Erklärungsgehalt hinsichtlich der Berufung externer Vorstandsvorsitzender aufweist. Auch ist zu berücksichtigen, dass mit zunehmender Grösse des Aufsichtsrates die Zahl der durch die Arbeitgeberseite zu besetzenden Mandate zunimmt. Hierbei liesse sich unterstellen, dass alleine durch die höhere Zahl vakanter Stellen die Berufung einer grösseren Anzahl externer Vorstände wahrscheinlicher ist. Um diesen möglichen Grösseneffekt zu überprüfen, wurde der Zusammenhang zwischen der Grösse des Aufsichtsrates und dem Anteil externer Vorstände an der Zahl der Arbeitgebervertreter im Aufsichtsrat in weiteren Schätzungen überprüft. Dabei zeigte sich ein auf dem zehn Prozent-Niveau signifikant positiver Zusammenhang nur für die Gruppe der anderen Vorstände. Für die Gruppe der Vorstandsvorsitzenden ergibt sich ein negativer und ebenfalls auf dem zehn Prozent-Niveau signifikanter Zusammenhang.

Das Argument einer Substitution von Managementexpertise wird ferner gestützt durch den Befund, dass externe Vorstände signifikant seltener in den Aufsichtsräten derjenigen Unternehmen aktiv sind, deren Vorstandsmitglieder selbst Mandate in externen Kontrollgremien ausüben. Der inverse Zusammenhang zwischen der Entsendung eigener Vorstände und dem Empfang fremder Vorstände im Aufsichtsrat deutet darauf hin, dass unterschiedliche Motive wie ein unternehmensspezifischer Bedarf an externer Kontroll- bzw. Beratungsexpertise oder die Entsendung von Anteilseignervertretern ursächlich für die jeweiligen Verflechtungsmuster zu sein scheinen. Die Analyse komplexerer Netzwerkstrukturen kann hierbei weiterführende Erkenntnisse liefern (siehe hierzu z. B. die Arbeiten von Beyer 1996 und Rank 2005).

In Anhang 1 sind ergänzend die Ergebnisse der Logit-Regressionen zusammengefasst. Hierbei wurden binäre abhängige Variablen verwendet, die den Wert eins annehmen, wenn mindestens ein entsprechendes Mandat im Aufsichtsrat vorhanden ist. Insgesamt liefern die alternativen Modellschätzungen qualitativ vergleichbare Ergebnisse, wobei gegenüber den Poisson-Regressionen tendenziell eine Abnahme der Signifikanz einzelner Koeffizienten festzustellen ist. Allerdings ergeben sich für keine der Variablen signifikante Änderungen in der Richtung des Zusammenhangs. Gleichwohl besteht im Unterschied zu Tabelle 5 ein signifikant positiver Zusammenhang zwischen dem Unternehmenserfolg und den abhängigen Variablen in allen Modellspezifikationen. Zudem ergibt sich ein positiver und signifikanter Zusammenhang zwischen dem Unternehmensalter und der Mitwirkung mindestens eines externen Vorstandsvorsitzenden im Aufsichtsrat. 


\subsection{Robustheit der Ergebnisse}

In den vergangenen Jahren liess sich eine fortschreitende Auflösung des sogenannten Systems der „Deutschland AG“ als Folge der Auflösung gegenseitiger Kapitalbeteiligungen beobachten (Höpner/Krempel 2004). Gleichzeitig legen empirische Befunde nahe, dass sich die Auflösung personeller Unternehmensverbindungen über Geschäftsführungs- und Kontrollgremien in einem weit geringeren Ausmass vollzieht. Dittmann et al. (2010) dokumentieren einen Rückgang gehaltener Kapitalbeteiligungen von Banken an Nicht-Finanzunternehmen von 4,1 Prozent auf 0,4 Prozent im Zeitraum 1994 bis 2005 und führen diese Entwicklung zu einem wesentlichen Teil auf die im Jahr 2002 in Kraft getretene Reform des Steuerrechts zurück, die den Verkauf von Unternehmensbeteiligungen steuerfrei stellte. Die Autoren stellen im gleichen Zeitraum einen vergleichsweise geringen Rückgang der Zahl von Bankenvertretern in den Aufsichtsräten von 9,6 auf 5,6 Prozent fest (ähnliche Befunde werden von der Monopolkommission 2006 berichtet). Übereinstimmend verdeutlicht Abbildung 1 eine schwach rückläufige Zahl externer Vorstände im Aufsichtsrat während des Zeitraums 1997 bis 2008.

Um zu überprüfen, inwieweit die Steuerrechtsreform des Jahres 2002 bzw. die erstmalige Veröffentlichung des Deutschen Corporate Governance Kodex im gleichen Jahr einen Einfluss auf Signifikanz und Richtung der im vorausgegangenen Abschnitt analysierten Koeffizienten ausübt, wird die Schätzung für die Zahl externer Vorstände im Aufsichtsrat als endogene Variable für die beiden Teilstichproben der Zeiträume vor und nach Inkrafttreten der Reform des Steuerrechts im Jahr 2002 wiederholt. Um einer zeitlichen Verzögerung zwischen der steuerlichen Befreiung von Veräusserungsgewinnen, die am 1. Januar 2002 wirksam wurde, und einer möglichen Neubesetzung von Aufsichtsratsgremien Rechnung zu tragen, wurde das Panel in die Zeiträume 1997 bis 2002 sowie 2003 bis 2008 aufgeteilt. Alternative Schätzungen für die Subsamples 1997 bis 2001 und 2002 bis 2008 führten zu qualitativ gleichen Ergebnissen. Zusätzlich wurde eine Dummyvariable als Indikator für den Zeitraum vor und nach den Reformen getestet, für den ein insignifikanter Koeffizient festgestellt wurde. Tabelle 6 fasst die Ergebnisse der Modellschätzungen zusammen.

Verglichen mit den Schätzergebnissen für den gesamten Untersuchungszeitraum in der Modellspezifikation (a) ergeben sich für die Spezifikationen (b) und (c) weitestgehend übereinstimmende Resultate. Signifikante Änderungen in der Richtung des Zusammenhangs können für keine der Variablen festgestellt werden. Der Unternehmenserfolg stellt sich wie auch die Grösse des Unternehmens als signifikant und positiv in den beiden Teilstichproben heraus. Externe Vorstände üben ab dem Jahr 2003 signifikant häufiger Mandate in älteren Unternehmen aus. Dies deutet darauf hin, dass ältere und etablierte Unternehmen, die über eine anzunehmende vergleichsweise hohe Zahl von Geschäftsbeziehungen zu anderen Unternehmen gekennzeichnet sind, weiterhin externe Vorstände als Aufseher beschäftigen und in geringerem Masse von der allgemeinen Auflösung personeller Verflechtungsbeziehungen betroffen sind. Der signifikant negative Zusammenhang zwischen der Diversifikation bzw. der positive Zusammenhang zwischen dem Streubesitz und der Zahl externer Vorstände im Aufsichtsrat zeigt sich nur für den Zeitraum bis 2002. Interessanterweise besteht zwischen dem Stimmrechtsanteil von Unternehmen aus dem Untersuchungskreis und dem Empfang externer Vorstände in beiden Teilstichproben ein signifikant positiver Zusammenhang. Der Befund deutet darauf hin, dass eine Reihe personeller 
Unternehmensverflechtungen auch weiterhin durch eine parallele Kapitalbeteiligung gekennzeichnet ist.

\begin{tabular}{|c|c|c|c|}
\hline & & & \\
\hline & (a) & (b) & (c) \\
\hline & \multicolumn{3}{|c|}{ Externe Vorstände im AR, davon im Zeitraum: } \\
\hline & $1997-2008$ & $1997-2002$ & $2003-2008$ \\
\hline \multirow[t]{2}{*}{ Return on Investment } & $1,738 * * *$ & $1,383 * * *$ & $3,144 * * *$ \\
\hline & $(4,88)$ & $(2,86)$ & $(3,14)$ \\
\hline \multirow[t]{2}{*}{ Log (Beschäftigte) } & $0,260 * \cdots$ & $0,205 * * *$ & $0,303 * *$ \\
\hline & $(7,15)$ & $(3,91)$ & $(6,45)$ \\
\hline \multirow[t]{2}{*}{ Alter des Unternehmens } & 0,001 & $-0,001$ & $0,002 * *$ \\
\hline & $(0,89)$ & $(-1,13)$ & $(2,32)$ \\
\hline \multirow[t]{2}{*}{ Diversifikation } & $-0,037 * * *$ & $-0,038 * *$ & $-0,028$ \\
\hline & $(-2,77)$ & $(-2,13)$ & $(-1,37)$ \\
\hline \multirow[t]{2}{*}{ Listung } & 0,013 & 0,062 & $-0,034$ \\
\hline & $(0,15)$ & $(0,56)$ & $(-0,29)$ \\
\hline \multirow[t]{2}{*}{ Streubesitz } & $0,004 * * *$ & $0,006 * * *$ & 0,001 \\
\hline & $(2,98)$ & $(3,76)$ & $(0,36)$ \\
\hline \multirow[t]{2}{*}{ Kapitalanteil Unternehmen } & $0,015 * * *$ & $0,017 * * *$ & $0,011 * * *$ \\
\hline & $(16,56)$ & $(15,22)$ & $(8,07)$ \\
\hline \multirow[t]{2}{*}{ Mitglieder Vorstand } & $-0,063 * * *$ & $-0,061 * * *$ & $-0,067 * * *$ \\
\hline & $(-5,39)$ & $(-3,63)$ & $(-4,10)$ \\
\hline \multirow[t]{2}{*}{ Mitglieder AR } & $0,030 * * *$ & 0,020 & $0,048 * * *$ \\
\hline & $(2,82)$ & $(1,49)$ & $(2,92)$ \\
\hline \multirow[t]{2}{*}{ Externe Mandate Vorstand } & $-0,076 * * *$ & $-0,073 * *$ & $-0,069 * *$ \\
\hline & $(-3,39)$ & $(-2,42)$ & $(-2,12)$ \\
\hline Beobachtungen & 604 & 304 & 300 \\
\hline Pseudo $\mathrm{R}^{2}$ & 0,263 & 0,269 & 0,276 \\
\hline Log Likelihood & $-627,909$ & $-323,436$ & $-295,378$ \\
\hline
\end{tabular}

Anmerkungen: Die Tabelle enthält Poisson-Regressionen zur Erklärung der Präsenz externer Vorstände im Aufsichtsrat. Als abhängige Variablen werden (a) die Zahl externer Vorstände im Aufsichtsrat im Zeitraum 1997 bis 2008 (vgl. Tabelle 5, Modell (a)), (b) die Zahl externer Vorstände im Aufsichtsrat in der Teilstichprobe 1997 bis 2002 und (c) die Zahl externer Vorstände im Aufsichtsrat in der Teilstichprobe 2003 bis 2008 herangezogen. Die exogenen Variablen fliessen mit den um eine Periode verzögerten Werten in die Schätzungen ein. In allen Schätzungen wurden Perioden- und Industriedummies verwendet. Berichtet werden die marginalen Effekte der einzelnen Koeffizienten mit den zugehörigen z-Werten in Klammern. Alle Modelle wurden mit robusten Standardfehlern geschätzt. ***, **, * signifikant auf dem $1 \%$, $5 \%-, 10 \%$-Niveau.

Tabelle 6: Schätzergebnisse für die Subsample 1997 bis 2002 und 2003 bis 2008 
Robust über die Zeit bleibt der signifikant negative Zusammenhang zwischen der Grösse des Vorstandsgremiums sowie der Zahl der externen Kontrollmandate der Vorstandsmitglieder und der Anzahl externer Vorstände im Aufsichtsrat. Der signifikant positive Einfluss der Grösse des Aufsichtsratsgremiums findet sich dagegen bei der Analyse der beiden Teilstichproben nur für das Zeitintervall ab 2003.

Anhang 2 fasst die Ergebnisse der Logit-Regressionen für die untersuchten Teilstichproben in den Spezifikationen (b) und (c) sowie für den gesamten Untersuchungszeitraum (Modell (a)) zusammen. Im Vergleich zu den Schätzungen in Tabelle 6 ergibt sich wiederum teilweise eine Abschwächung der Signifikanz einzelner Koeffizienten. Dies betrifft insbesondere den Erklärungsgehalt der Variablen zur Diversifikation, zum Anteil des Streubesitzes sowie zur Grösse des Aufsichtsratsgremiums. Signifikante Änderungen in der Richtung des Zusammenhangs sind für keine der Variablen gegenüber den Poisson-Modellen festzustellen.

Zusammenfassend legen die Befunde nahe, dass die Berufung externer Mandatsträger in den Aufsichtsrat auch nach den skizzierten Veränderungen des institutionellen Rahmens weiterhin eine gängige Unternehmenspraxis darstellt. Es ist weiterhin zu vermuten, dass die Veröffentlichung des Deutschen Corporate Governance Kodex zumindest während des betrachteten Untersuchungszeitraumes nur einen vergleichbar geringen Einfluss auf die Zahl der Mehrfachmandatsträger in deutschen Grossunternehmen ausgeübt hat.

\section{Zusammenfassung der Ergebnisse und Forschungsausblick}

Vorstände deutscher Grossunternehmen werden regelmässig als externe Kontrolleure in den Aufsichtsräten anderer Grossunternehmen nachgefragt. Im Rahmen dieser Arbeit wurden unternehmensspezifische Variablen identifiziert, anhand derer die Anzahl externer Führungskräfte in deutschen Aufsichtsräten erklärt werden kann. Die erwarteten Zusammenhänge wurden mittels eines Datensatzes zu deutschen Grossunternehmen für den Zeitraum 1997 bis 2008 empirisch untersucht. Die Ergebnisse verdeutlichen, dass neben dem Unternehmenserfolg und der Grösse eine Reihe weiterer Faktoren zur Komplexität und Kapitalstruktur einen signifikanten Erklärungsgehalt aufweist.

Getrennte Schätzungen für die Anzahl externer Vorstandsvorsitzender und gewöhnlicher Vorstandsmitglieder im Aufsichtsrat heben die herausragende Stellung von Vorstandsvorsitzenden als externe Kontrolleure hervor. In Übereinstimmung mit der Argumentation von Fablenbrach et al. (2010a), die in einer empirischen Untersuchung zu USamerikanischen Unternehmen die Motive der Berufung externer CEOs in das Board of Directors diskutieren und empirisch analysieren, finden sich Hinweise, dass Vorstandsvorsitzende häufiger Mandate in prestigeträchtigeren Unternehmen anstreben bzw. häufiger in entsprechende Unternehmen berufen werden. Ein positiver Einfluss des Unternehmenserfolgs und der Börsenlistung zeigt sich ausschliesslich für die Gruppe der Vorstandsvorsitzenden im Aufsichtsrat. Darüber hinaus stellt aber auch die Berufung gewöhnlicher Vorstandsmitglieder in die Aufsichtsratsgremien deutscher Grossunternehmen ein häufiges Verflechtungsmuster dar, das bislang in der empirischen Corporate Governance-Forschung nur unzureichend Beachtung gefunden hat. Für 20,7 Prozent der Unternehmen in der Stichprobe konnte mindestens ein externer Vorstand, der nicht die Position des Vorsitzenden ausübt, im Aufsichtsrat identifiziert werden. Die durchschnittliche Zahl externer Vorstände im Aufsichtsrat beträgt 0,39. Für künftige Forschungsarbeiten wäre es von besonderem Interesse, die Position des jeweiligen Vorstandes in dem entsendenden Unterneh- 
men oder auch seine bisherigen Berufsstationen auszuwerten, um die Motive für die Ernennung tiefergehend beleuchten zu können. Von Interesse wäre zudem die Berücksichtigung weiterer Personengruppen seitens der Arbeitgebervertreter im Aufsichtsrat mit spezifischen und knappen Kompetenzen wie z. B. unabhängige Mandatsträger aus dem Bereich der Wissenschaft, denen ein zunehmendes Gewicht bei der Besetzung von Aufsichtsratsposition beizumessen ist. $^{6}$

Das vorliegende Untersuchungsdesign beschränkt sich zudem auf die Erklärung bestehender Mandate und erlaubt keine Interpretation kausaler Zusammenhänge hinsichtlich der Wirkungen von externen Vorstandsberufungen auf den Unternehmenserfolg oder die Kontroll- und Beratungsqualität des Aufsichtsratsgremiums. Als interessanter Ansatzpunkt für weiterführende Untersuchungen ist in diesem Zusammenhang insbesondere neben der Analyse unternehmensrelevanter Variablen zum Zeitpunkt der Aufsichtsratsberufung auch die Untersuchung von Faktoren zu nennen, anhand derer das Ausscheiden eines externen Vorstands aus dem Kontrollgremium erklärt werden kann. Balsmeier et al. (2010b) analysieren für ein Sample von DAX30-Unternehmen im Zeitraum 2001 bis 2005 den Einfluss verschiedener Kontrollvariablen auf die Fluktuation von Vorstandsmitgliedern (siehe auch Gregory-Smith et al. 2009 zu einer Studie zu britischen Unternehmen). Balsmeier et al. (2010b) zeigen unter anderem, dass Vorstände anderer DAX-Unternehmen im Aufsichtsrat die Austrittswahrscheinlichkeit eigener Vorstände signifikant erhöhen. Auf Grundlage eines vergleichbaren Datensatzes untersuchen Balsmeier et al. (2010a) in einer weiteren Studie die Hazardraten von Arbeitgeber- und Arbeitnehmervertretern im Aufsichtsrat. Die Autoren zeigen neben einer Reihe weiterer Befunde, dass der Unternehmenserfolg die Fluktuation von Arbeitgebervertretern im Aufsichtsrat erhöht, während die Höhe der Vergütung der Aufsichtsratsmitglieder einen negativen Einfluss auf die Hazardrate ausübt. Eine tiefergehende Unterscheidung verschiedener Mandatsträger stellt hierbei einen Ansatz für weiterführende Untersuchungen dar. Auch die Studie von Fablenbrach et al. (2010b) zu US-amerikanischen Unternehmen liefert eine Reihe interessanter Befunde. Die Autoren finden unter anderem Anhaltspunkte dafür, dass externe Vorstände dann aus dem Board ausscheiden, wenn sie erwarten, dass die erwartete Rendite in den Folgeperioden sinkt.

Abschliessend zeigen die Ergebnisse der empirischen Untersuchung, dass personelle Verflechtungen zwischen Unternehmen, die nicht schwerpunktmässig dem Kredit- oder Versicherungsgewerbe zuzuordnen sind, auch nach Inkrafttreten der Reform des Steuerrechts und der Veröffentlichung des Corporate Governance Kodex im Jahr 2002 ein relevantes Verflechtungsmuster darstellen. In diesem Zusammenhang stellt die tiefergehende Analyse der Motive für personelle Verflechtungen zwischen Industrie-, Handels- und Dienstleistungsunternehmen, denen offensichtlich spezifische Anreize und Mechanismen zugrundeliegen, einen interessanten Ansatz für weiterführende Fragestellungen dar.

6 Der Deutsche Corporate Governance Kodex sieht in seiner aktuellen Fassung vom 26. Mai 2010 unter Ziffer 5.4.1 vor, dass hinsichtlich der Zusammensetzung des Aufsichtsrates auf Vielfalt geachtet werden sollte; der Kodex konkretisiert dieses Ziel jedoch explizit nur in Bezug auf eine angemessene Beteiligung von Frauen. 
Anhang
(a)
(b)
(c)

\begin{tabular}{|c|c|c|c|}
\hline & $\begin{array}{l}\text { Dummy externe } \\
\text { Vorstände im AR }\end{array}$ & $\begin{array}{l}\text { Dummy externe } \\
\text { Vorstände (exkl. } \\
\text { Vorsitz) im AR }\end{array}$ & $\begin{array}{c}\text { Dummy externe } \\
\text { Vorstandsvorsitzende } \\
\text { im AR }\end{array}$ \\
\hline \multirow[t]{2}{*}{ Return on Investment } & $5,243 * * *$ & $5,846 * *$ & $4,774 * * *$ \\
\hline & $(2,88)$ & $(2,51)$ & $(2,62)$ \\
\hline \multirow[t]{2}{*}{ Log (Beschäftigte) } & $0,628 * *$ & $0,538 * *$ & $0,554 * * *$ \\
\hline & $(4,93)$ & $(3,28)$ & $(4,46)$ \\
\hline \multirow[t]{2}{*}{ Alter des Unternehmens } & 0,003 & 0,002 & $0,004 * *$ \\
\hline & $(1,26)$ & $(0,79)$ & $(2,03)$ \\
\hline \multirow[t]{2}{*}{ Diversifikation } & $-0,023$ & $-0,336 * * *$ & $0,198 * * *$ \\
\hline & $(-0,32)$ & $(-3,69)$ & $(2,91)$ \\
\hline \multirow[t]{2}{*}{ Listung } & 0,456 & $-0,466$ & $1,220 * * *$ \\
\hline & $(1,58)$ & $(-1,19)$ & $(4,18)$ \\
\hline \multirow[t]{2}{*}{ Streubesitz } & $0,012 * *$ & 0,005 & 0,006 \\
\hline & $(2,51)$ & $(0,92)$ & $(1,38)$ \\
\hline \multirow[t]{2}{*}{ Kapitalanteil Unternehmen } & $0,040 * *$ & $0,043 * * *$ & $0,014 * * *$ \\
\hline & $(5,96)$ & $(6,88)$ & $(3,07)$ \\
\hline \multirow[t]{2}{*}{ Mitglieder Vorstand } & $-0,131 * * *$ & $-0,219 * * *$ & $-0,081^{\#}$ \\
\hline & $(-2,81)$ & $(-3,11)$ & $(-1,75)$ \\
\hline \multirow[t]{2}{*}{ Mitglieder AR } & 0,015 & $0,190 * * *$ & $-0,028$ \\
\hline & $(0,52)$ & $(3,89)$ & $(-0,91)$ \\
\hline \multirow[t]{2}{*}{ Externe Mandate Vorstand } & $-0,212 * * *$ & $-0,140$ & $-0,245 * * *$ \\
\hline & $(-2,60)$ & $(-1,25)$ & $(-3,02)$ \\
\hline \multirow[t]{2}{*}{ Konstante } & $-7,392 * * *$ & $-9,169 * * *$ & $-7,661 * * *$ \\
\hline & $(-5,62)$ & $(-5,03)$ & $(-5,84)$ \\
\hline Beobachtungen & 604 & 604 & 604 \\
\hline Pseudo $\mathrm{R}^{2}$ & 0,210 & 0,247 & 0,162 \\
\hline$\underline{\text { Log Likelihood }}$ & $-330,593$ & $-231,923$ & $-341,887$ \\
\hline
\end{tabular}

Anmerkungen: Die Tabelle enthält Logit-Regressionen zur Erklärung der Präsenz externer Vorstände im Aufsichtsrat. Als endogene Variable wird ein Dummy verwendet, der den Wert eins annimmt, wenn mindestens (a) ein externer Vorstand, (b) ein externer Vorstand (exkl. Vorstandsvorsitzende) und (c) ein externer Vorstandsvorsitzender ein Mandat im Aufsichtsrat ausübt. Die exogenen Variablen fliessen mit den um eine Periode verzögerten Werten in die Schätzungen ein. In allen Schätzungen wurden Perioden- und Industriedummies verwendet. Berichtet werden die einzelnen Koeffizienten mit den zugehörigen z-Werten in Klammern. ***, **, \#: signifikant auf dem $1 \%-, 5 \%-, 10 \%$-Niveau.

Anhang 1: Bestimmungsfaktoren für die Präsenz externer Vorstände im Aufsichtsrat 


\begin{tabular}{|c|c|c|c|}
\hline & (a) & (b) & (c) \\
\hline & \multicolumn{3}{|c|}{ Dummy externe Vorstände im AR, davon im Zeitraum: } \\
\hline & $1997-2008$ & $1997-2002$ & $2003-2008$ \\
\hline \multirow[t]{2}{*}{ Return on Investment } & $5,243 * *$ & $3,822 * *$ & $8,794 * *$ \\
\hline & $(2,88)$ & $(1,97)$ & $(2,27)$ \\
\hline \multirow[t]{2}{*}{ Log (Beschäftigte) } & $0,628 * * *$ & $0,592 * * *$ & $0,862 * * *$ \\
\hline & $(4,93)$ & $(3,18)$ & $(4,14)$ \\
\hline \multirow[t]{2}{*}{ Alter des Unternehmens } & 0,003 & $-0,000$ & 0,005 \\
\hline & $(1,26)$ & $(-0,04)$ & $(1,56)$ \\
\hline \multirow[t]{2}{*}{ Diversifikation } & $-0,023$ & 0,094 & $-0,206^{\#}$ \\
\hline & $(-0,32)$ & $(0,92)$ & $(-1,75)$ \\
\hline \multirow[t]{2}{*}{ Listung } & 0,456 & $0,738^{\#}$ & 0,347 \\
\hline & $(1,58)$ & $(1,85)$ & $(0,72)$ \\
\hline \multirow[t]{2}{*}{ Streubesitz } & $0,012 * *$ & 0,006 & $0,014^{\#}$ \\
\hline & $(2,51)$ & $(0,87)$ & $(1,94)$ \\
\hline \multirow[t]{2}{*}{ Kapitalanteil Unternehmen } & $0,040 * * *$ & $0,062 * * *$ & $0,022 * * *$ \\
\hline & $(5,96)$ & $(5,35)$ & $(2,76)$ \\
\hline \multirow[t]{2}{*}{ Mitglieder Vorstand } & $-0,131 * * *$ & $-0,095$ & $-0,230 * * *$ \\
\hline & $(-2,81)$ & $(-1,52)$ & $(-2,68)$ \\
\hline \multirow[t]{2}{*}{ Mitglieder AR } & 0,015 & $-0,022$ & $0,092^{\#}$ \\
\hline & $(0,52)$ & $(-0,59)$ & $(1,69)$ \\
\hline \multirow[t]{2}{*}{ Externe Mandate Vorstand } & $-0,212 * * *$ & $-0,225 * *$ & $-0,152$ \\
\hline & $(-2,60)$ & $(-2,08)$ & $(-1,12)$ \\
\hline \multirow[t]{2}{*}{ Konstante } & $-7,392 * * *$ & $-6,787 * * *$ & $-10,575 * * *$ \\
\hline & $(-5,62)$ & $(-3,70)$ & $(-4,85)$ \\
\hline Beobachtungen & 604 & 304 & 300 \\
\hline Pseudo $\mathrm{R}^{2}$ & 0,210 & 0,240 & 0,240 \\
\hline Log Likelihood & $-330,593$ & $-158,618$ & $-157,885$ \\
\hline
\end{tabular}

Anmerkungen: Die Tabelle enthält Logit-Regressionen zur Erklärung der Präsenz externer Vorstände im Aufsichtsrat. Als endogene Variable wird ein Dummy verwendet, der den Wert eins annimmt, wenn mindestens (a) ein externer Vorstand im Zeitraum 1997 bis 2008 (vgl. Anhang 1, Modell (a)), (b) ein externer Vorstand im Zeitraum 1997 bis 2002 und (c) ein externer Vorstand im Zeitraum 2003 bis 2008 ein Mandat im Aufsichtsrat ausübt. Die exogenen Variablen fliessen mit den um eine Periode verzögerten Werten in die Schätzungen ein. In allen Schätzungen wurden Perioden- und Industriedummies verwendet. Berichtet werden die einzelnen Koeffizienten mit den zugehörigen $z$-Werten in Klammern. ${ }^{* * *}$, **, *: signifikant auf dem $1 \%-, 5 \%-, 10 \%$-Niveau.

Anhang 2: Schätzergebnisse für die Subsample 1997 bis 2002 und 2003 bis 2008 


\begin{tabular}{|c|c|c|c|}
\hline & (a) & (b) & (c) \\
\hline & $\begin{array}{c}\text { Externe Vorstände } \\
\text { im AR }\end{array}$ & $\begin{array}{c}\text { Externe Vorstände } \\
\text { (exkl. Vorsitz) } \\
\text { im AR }\end{array}$ & $\begin{array}{l}\text { Externe Vorstands- } \\
\text { vorsitzende im AR }\end{array}$ \\
\hline \multirow[t]{2}{*}{ Return on Investment } & $2,175 * *$ & $0,551 \%$ & $1,248 * * *$ \\
\hline & $(7,42)$ & $(2,39)$ & $(6,16)$ \\
\hline \multirow[t]{2}{*}{ Log (Beschäftigte) } & $0,276 * * *$ & $0,042 * *$ & $0,221 * * *$ \\
\hline & $(6,46)$ & $(2,30)$ & $(6,62)$ \\
\hline \multirow[t]{2}{*}{ Alter des Unternehmens } & 0,001 & 0,000 & 0,001 \\
\hline & $(1,23)$ & $(0,91)$ & $(1,56)$ \\
\hline \multirow[t]{2}{*}{ Diversifikation } & $-0,034 * *$ & $-0,027 * * *$ & 0,020 \\
\hline & $(-2,24)$ & $(-4,40)$ & $(1,50)$ \\
\hline \multirow[t]{2}{*}{ Listung } & 0,040 & $-0,026$ & $0,164 * *$ \\
\hline & $(0,43)$ & $(-0,62)$ & $(2,21)$ \\
\hline \multirow[t]{2}{*}{ Streubesitz } & $0,003 * *$ & 0,000 & $0,002 *$ \\
\hline & $(2,56)$ & $(0,63)$ & $(1,93)$ \\
\hline \multirow[t]{2}{*}{ Kapitalanteil Unternehmen } & $0,016 * * *$ & $0,005 * *$ & $0,004 * * *$ \\
\hline & $(15,68)$ & $(7,76)$ & $(4,21)$ \\
\hline \multirow[t]{2}{*}{ Mitglieder Vorstand } & $-0,060 * * *$ & $-0,022 * * *$ & $-0,036 * * *$ \\
\hline & $(-4,75)$ & $(-3,32)$ & $(-3,45)$ \\
\hline \multirow[t]{2}{*}{ Mitglieder AR } & $0,030 * *$ & $0,025 * * *$ & $-0,001$ \\
\hline & $(2,37)$ & $(4,45)$ & $(-0,10)$ \\
\hline \multirow[t]{2}{*}{ Externe Mandate Vorstand } & $-0,076 * * *$ & $-0,012$ & $-0,067 * * *$ \\
\hline & $(-3,08)$ & $(-1,04)$ & $(-3,43)$ \\
\hline Beobachtungen & 517 & 517 & 517 \\
\hline Pseudo R2 & 0,262 & 0,433 & 0,117 \\
\hline Log Likelihood & $-543,515$ & $-282,947$ & $-445,550$ \\
\hline
\end{tabular}

Anmerkungen: Die Tabelle enthält Poisson-Regressionen zur Erklärung der Präsenz externer Vorstände im Aufsichtsrat. Als abhängige Variablen werden (a) die Zahl externer Vorstände im Aufsichtsrat insgesamt, (b) die Zahl externer Vorstände (exkl. Vorstandsvorsitzende) und (c) die Zahl externer Vorstandsvorsitzender im Aufsichtsrat herangezogen. Die exogenen Variablen fliessen mit den um zwei Perioden verzögerten Werten in die Schätzungen ein. In allen Schätzungen wurden Perioden- und Industriedummies verwendet. Berichtet werden die marginalen Effekte der einzelnen Koeffizienten mit den zugehörigen z-Werten in Klammern. Alle Modelle wurden mit robusten Standardfehlern geschätzt. ***, **, ${ }^{\#}$ : signifikant auf dem $1 \%-, 5 \%-, 10 \%$-Niveau.

Anhang 3: Bestimmungsfaktoren für die Präsenz externer Vorstände im Aufsichtsrat 


\begin{tabular}{|c|c|c|c|}
\hline & (a) & (b) & (c) \\
\hline & $\begin{array}{c}\text { Externe Vorstände } \\
\text { im AR }\end{array}$ & $\begin{array}{c}\text { Externe Vorstände } \\
\text { (exkl. Vorsitz) } \\
\text { im AR }\end{array}$ & $\begin{array}{l}\text { Externe Vorstands- } \\
\text { vorsitzende im AR }\end{array}$ \\
\hline \multirow[t]{2}{*}{ Return on Investment } & $1,800 * * *$ & $-0,236$ & $1,241 * * *$ \\
\hline & $(5,45)$ & $(-0,42)$ & $(6,00)$ \\
\hline \multirow[t]{2}{*}{ Log (Beschäftigte) } & $0,319 * * *$ & $0,037^{*}$ & $0,256 * *$ \\
\hline & $(7,22)$ & $(1,68)$ & $(7,67)$ \\
\hline \multirow[t]{2}{*}{ Alter des Unternehmens } & 0,001 & 0,000 & $0,001 * *$ \\
\hline & $(1,53)$ & $(0,33)$ & $(2,56)$ \\
\hline \multirow[t]{2}{*}{ Diversifikation } & $-0,037 * *$ & $-0,029 * * *$ & 0,014 \\
\hline & $(-2,31)$ & $(-3,89)$ & $(1,08)$ \\
\hline \multirow[t]{2}{*}{ Listung } & $-0,032$ & $-0,080$ & $0,153 *$ \\
\hline & $(-0,28)$ & $(-1,39)$ & $(1,85)$ \\
\hline \multirow[t]{2}{*}{ Streubesitz } & $0,004 * * *$ & 0,001 & 0,002 \\
\hline & $(2,68)$ & $(1,43)$ & $(1,52)$ \\
\hline \multirow[t]{2}{*}{ Kapitalanteil Unternehmen } & $0,015 * *$ & $0,005 * * *$ & $0,004 * * *$ \\
\hline & $(14,22)$ & $(6,66)$ & $(3,84)$ \\
\hline \multirow[t]{2}{*}{ Mitglieder Vorstand } & $-0,069 * *$ & $-0,020 * *$ & $-0,045 * *$ \\
\hline & $(-4,92)$ & $(-2,55)$ & $(-4,18)$ \\
\hline \multirow[t]{2}{*}{ Mitglieder AR } & $0,023 *$ & $0,023 * *$ & $-0,002$ \\
\hline & $(1,83)$ & $(3,44)$ & $(-0,21)$ \\
\hline \multirow[t]{2}{*}{ Externe Mandate Vorstand } & $-0,093 * * *$ & $-0,014$ & $-0,075 * *$ \\
\hline & $(-3,42)$ & $(-1,14)$ & $(-3,55)$ \\
\hline Beobachtungen & 443 & 443 & 443 \\
\hline Pseudo R2 & 0,267 & 0,423 & 0,131 \\
\hline Log Likelihood & $-468,688$ & $-252,139$ & $-376,809$ \\
\hline
\end{tabular}

Anmerkungen: Die Tabelle enthält Poisson-Regressionen zur Erklärung der Präsenz externer Vorstände im Aufsichtsrat. Als abhängige Variablen werden (a) die Zahl externer Vorstände im Aufsichtsrat insgesamt, (b) die Zahl externer Vorstände (exkl. Vorstandsvorsitzende) und (c) die Zahl externer Vorstandsvorsitzender im Aufsichtsrat herangezogen. Die exogenen Variablen fliessen mit den um drei Perioden verzögerten Werten in die Schätzungen ein. In allen Schätzungen wurden Perioden- und Industriedummies verwendet. Berichtet werden die marginalen Effekte der einzelnen Koeffizienten mit den zugehörigen z-Werten in Klammern. Alle Modelle wurden mit robusten Standardfehlern geschätzt. ${ }^{* * *}, * *$, : signifikant auf dem $1 \%-, 5 \%-, 10 \%$-Niveau.

Anhang 4: Bestimmungsfaktoren für die Präsenz externer Vorstände im Aufsichtsrat 


\section{Literaturhinweise}

Adams, R.B./Ferreira, D. (2007): A Theory of Friendly Boards, in: Journal of Finance, Jg. 62, Nr. 1, S. 217-250.

Adams, R.B./Hermalin, B.E./Weisbach, M.S. (2010): The Role of Boards of Directors in Corporate Governance: A Conceptual Framework \& Survey, in: Journal of Economic Literature, Jg. 48, Nr. 1, S. 58-107.

Agrawal, A./Knoeber, C.R. (2001): Do Some Outside Directors Play a Political Role, in: Journal of Law and Economics, Jg. 44, Nr. 1, S. 179-198.

Andres, C./Lehmann, M. (2010): Is Busy Really Busy? Board Governance Revisited, SSRN Working Paper, im Internet unter: http://ssrn.com/abstract=1569531.

Balsmeier, B./Dilger, A./Lingens, J. (2010a): Hazard Rates and Personal Interdependencies in Supervisory Boards of DAX-Companies, German Economic Assocation of Business Administration (GEABA), Discussion Paper 10-19, im Internet unter: http://whu.edu/geaba/Papers/2010/ DP_10-19.pdf.

Balsmeier, B./Dilger, A./Lingens, J. (2010b): Fluktuation von Vorständen und personelle Verflechtungen in DAX-Unternehmen, in: Zeitschrift für Betriebswirtschaft, Jg. 80, Nr. 10, S. 1007-1026.

Balsmeier, B./Peters, H. (2009): Personelle Unternehmensverflechtung und Vorstandsgehälter, in: Zeitschrift für Betriebswirtschaft, Jg. 79, Nr.9, S. 967-984.

Balsmeier, B./Buchwald, A. (2011): Motive der Ausübung externer Kontrollmandate durch Vorstandsvorsitzende in deutschen Großunternehmen, in: Die Betriebswirtschaft, Jg. 71, Nr. 2, S. 101-119.

Balsmeier, B./Buchwald, A./Peters, H. (2010): Auswirkungen von Mehrfachmandaten deutscher Vorstands- und Aufsichtsratsvorsitzender auf den Unternehmenserfolg, in: Jahrbücher für Nationalökonomie und Statistik, Jg. 230, Nr. 5, S. 547-570.

Barnea, A./Guedj, I. (2009): Director Networks, EFA 2007 Ljubljana Meetings Paper, im Internet unter: http://papers.ssrn.com/sol3/papers.cfm?abstract_id=966555.

Bebchuk, L.A./Cohen, A./Ferrell, A. (2009): What Matters in Corporate Governance, in: Review of Financial Studies, Jg. 22, Nr. 2, S. 783-827.

Becht, M./Bolton, P./Röell, A. (2002): Corporate Governance and Control, ecgi Finance Working Paper, No. 02/2002, Brüssel, im Internet unter: http://unpan1.un.org/intradoc/groups/public/ documents/apcity/unpan033582.pdf.

Beetz, A. (2005): Vergleich der deutschen Corporate Governance mit den Regelungen in den USA, in: Forum: Neues Wirtschaftsrecht NWiR, Jg. 7, S. 1-23.

Beyer, J. (1996): Governance Structures - Unternehmensverflechtungen und Unternehmenserfolg in Deutschland, in: Zeitschrift für Betriebswirtschaft, Ergänzungsheft 3/1996, S. 79-101.

Biebler, H./Ortmann, R. (1985): Personelle Verflechtungen zwischen Unternehmen: Ergebnisse einer Interviewserie bei Vorstands- und Aufsichtsratsmitgliedern großer deutscher Unternehmen, in: Die Betriebswirtschaft, Jg. 45, Nr. 1, S. 4-18.

Boone, A.L./Field, L.C./Karpoff, J.M./Raheja, C.G. (2007): The Determinants of Corporate Board Size and Independence: An Empirical Analysis, in: Journal of Financial Economics, Jg. 85, Nr. 1, S. 65-101.

Booth, J.R./Deli, D.N. (1996): Factors Affecting the Number of Outside Directorships Held by CEOs, in: Journal of Financial Economics, Jg. 40, Nr. 1, S. 81-104. 
Cameron, A.C./Trivedi, P.K. (1998): Regression Analysis of Count Data, Cambridge UK.

Cameron, A.C./Trivedi, P.K. (2010): Microeconometrics using Stata, College Station, Texas.

Coles, J.L./Daniel, N.D./Naveen, L. (2008): Boards: Does one size fit it all, in: Journal of Financial Economics, Jg. 87, Nr. 2, S. 329-356.

Conyon, M.J./Read, L.E. (2006): A Model of the Supply of Executives for Outside Directorships, in: Journal of Corporate Finance, Jg. 12, Nr. 3, S. 645-659.

Dittmann, I./Maug, E./Schneider, C. (2010): Bankers on the Boards of German Firms: What They Do, What They are Worth, and Why They are (Still) There, in: Review of Finance, Jg. 14, Nr. 1, S. 35-71.

Entorf, H./Gattung, F./Möbert, J./Pahlke, I. (2009): Aufsichtsratsverflechtungen und ihr Einfluss auf die Vorstandsbezüge von DAX-Unternehmen, in: Zeitschrift für Betriebswirtschaft, Jg. 79, Nr. 11, S. 1113-1141.

Fablenbrach, R./Low, A./Stulz, R.M. (2010a): Why Do Firms Appoint CEOs as Outside Directors, in: Journal of Financial Economics, Jg. 97, Nr. 1, S. 12-32.

Fablenbrach, R./Low, A./Stulz, R.M. (2010b): The Dark Side of Outside Directors: Do They Quit When They are Most Needed?, NBER Working Paper, No. 15917, Cambridge, Mass, im Internet unter: http://www.nber.org/papers/w15917.

Faleye, O. (2003): Are Large Boards Poor Monitors? Evidence from CEO Turnover, SSRN Working Paper, im Internet unter: http://papers.ssrn.com/sol3/papers.cfm?abstract_id=498285.

Fama, E.F./Jensen, M.C. (1983): Separation of Ownership and Control, in: Journal of Law and Economics, Jg. 26, Nr. 2, S. 301-325.

Fauver, L./Fuerst, M.E. (2006): Does Good Corporate Governance Include Employee Representation? Evidence from German Corporate Boards, in: Journal of Financial Economics, Jg. 82, Nr. 3, S. 673-710.

Fich, E.M. (2005): Are Some Outside Directors Better than Others? Evidence from Director Appointments by Fortune 1000 Firms, in: Journal of Business, Jg. 78, Nr. 5, S. 1943-1971.

Fich, E.M./Shivdasani, A. (2006): Are Busy Boards Effective Monitors, in: Journal of Finance, Jg. 61, Nr. 2, S. 689-724.

Gregory-Smith, I./Thompson, S./Wright, P.W. (2009): Fired or Retired? A Competing Risks Analysis of Chief Executive Turnover, in: The Economic Journal, Jg. 119, Nr. 3, S. 463-481.

Güner, A.B./Malmendier, U./Tate, G. (2008): Financial Expertise of Directors, in: Journal of Financial Economics, Jg. 88, Nr. 2, S. 323-354.

Heinze, T. (2004): Dynamics in the German System of Corporate Governance? Empirical Findings Regarding Interlocking Directorates, in: Economy and Society, Jg. 33, Nr. 2, S. 218-238.

Hermalin, B.E. (2005): Trends in Corporate Governance, in: Journal of Finance, Jg. 60, Nr. 5, S. 2351-2384.

Höpner, M./Müllenborn, T. (2010): Mitbestimmung im Unternehmensvergleich: Ein Konzept zur Messung des Einflusspotentials der Arbeitnehmervertreter im mitbestimmten Aufsichtsrat, in: Industrielle Beziehungen, Jg. 17, Nr. 1, S. 7-29.

Höpner, M./Krempel, L. (2004): The Politics of the German Company Network, in: Competition \& Change, Jg. 8, Nr. 4, S. 339-356.

Jensen, M.C./Meckling, W.H. (1976): Theory of the Firm: Managerial Behavior, Agency Costs and Ownership Structure, in: Journal of Financial Economics, Jg. 3, Nr. 4, S. 305-360.

Johnson, P.S/Apps, R. (1979): Interlocking Directorates Among the UK's Largest Companies, in: Antitrust Bulletin, Jg. 24, Nr. 3, S. 357-369. 
Kaplan, S.N. (1994): Top Executives Turnover and Firm Performance in Germany, in: Journal of Law, Economics, \& Organization, Jg. 10, Nr. 1, S. 142-159.

Linck, J.S./Netter, J.M./Yang, T. (2009): The Effects and Unintended Consequences of the SarbanesOxley Act on the Supply and Demand for Directors, in: Review of Financial Studies, Jg. 22, Nr. 8, S. 3287-3328.

Linck, J.S./Netter, J.M./Yang, T. (2008): The Determinants of Board Structure, in: Journal of Financial Economics, Jg. 87, Nr. 2, S. 308-328.

Mace, M.L. (1986): Directors: Myth and Reality, Boston, MA.

Miwa, Y./Ramseyer, J.M. (2005): Who Appoints Them, What Do They Do? Evidence on Outside Directors from Japan, in: Journal of Economics \& Management Strategy, Jg. 14, Nr. 2, S. 299-337.

Mizruchi, M.S. (1996): What Do Interlocks Do? An Analysis, Critique, and Assessment of Research on Interlocking Directorates, in: Annual Review of Sociology, Jg. 22, Nr. 1, S. 271-298.

Monopolkommission (2006): Mehr Wettbewerb auch im Dienstleistungssektor!: Hauptgutachten 2004/2005, Baden-Baden.

Monopolkommission (2010): Mehr Wettbewerb, wenig Ausnahmen: Hauptgutachten 2008/2009, Baden-Baden.

Noll, B./Volkert, J./Zuber, N. (2011): Managermärkte: Wettbewerb und Zugangsbeschränkungen: Eine institutionen- und sozioökonomische Perspektive, Baden-Baden.

Pfannschmidt, A. (1995): Mehrfachmandate in deutschen Unternehmen. Ökonomischer Erklärungsansatz, in: Zeitschrift für Betriebswirtschaft, Jg. 65, Nr. 2, S. 177-203.

Pfeffer, J. (1972): Size and Composition of Corporate Boards of Directors: The Organization and its Environment, in: Administrative Science Quarterly, Jg. 17, Nr. 2, S. 218-228.

Pfeffer, J. (1992): A Resource Dependence Perspective on Intercorporate Relations, in: Mizruchi, M.S./Schwartz, M. (Hrsg.): Intercorporate Relations. The Structural Analysis of Business, Cambridge, S. 25-55.

Rank, O. (2005): Interdependenzen in den Governance-Strukturen deutscher Großunternehmen, in: Zeitschrift für Betriebswirtschaft, Jg. 75, Nr. 1, S. 15-41.

Rank, O. (2006): Dynamics of the German Governance Network, in: Zeitschrift für Betriebswirtschaft, Ergänzungsheft 5/2006, S. 75-98.

Ringleb, H.-M./Kremer, T./Lutter, M./ von Werder, A. (2010): Kommentar zum Deutschen Corporate Governance Kodex: Kodex-Kommentar, München.

Schönwitz, D./Weber, H.-J. (1980): Personelle Verflechtungen zwischen Unternehmen. Eine wettbewerbspolitische Analyse, in: Zeitschrift für die gesamte Staatswissenschaft, Jg. 136, Nr. 1, S. $98-112$.

Schreyögg, G./Papenheim-Tockhorn, H. (1995): Dient der Aufsichtsrat dem Aufbau zwischenbetrieblicher Kooperationsbeziehungen. Eine Längsschnittstudie zur Rekonstitution „gebrochener Verflechtungen“ zwischen deutschen Kapitalgesellschaften, in: Zeitschrift für Betriebswirtschaft, Jg. 65, Nr. 2, S. 205-230.

Shivdasani, A./Yermack, D. (1999): CEO Involvement in the Selection of New Board Members: An Empirical Analysis, in: Journal of Finance, Jg. 54, Nr. 5, S. 1829-1853.

Shleifer, A./Vishny, R.W. (1997): A Survey of Corporate Governance, in: Journal of Finance, Jg. 52, Nr. 2, S. 737-783.

Weisbach, M.S. (1988): Outside Directors and CEO Turnover, in: Journal of Financial Economics, Jg. 20, Nr. 1/2, S. 431-460. 
Windolf, P. (1997): Eigentum und Herrschaft: Elitenetzwerke in Deutschland und Großbritannien, in: Leviathan, Jg. 25, Nr. 1, S. 76-106.

Winkelmann, R. (2008): Econometric Analysis of Count Data, Berlin, Heidelberg.

Yermack, D. (1996): Higher Market Valuation of Companies with a Small Board of Directors, in: Journal of Financial Economics, Jg. 40, Nr. 2, S. 185-211.

Zimmermann, S. (2010): Interne versus externe Rekrutierung von Vorständen in deutschen Aktiengesellschaften, in: Schmalenbachs Zeitschrift für betriebswirtschaftliche Forschung, Jg. 62, S. 160-202.

Achim Buchwald, Dr., ist Senior Economist bei der Monopolkommission, Bonn und ehem. Doktorand am Institut für Organisationsökonomik an der Universität Münster.

Anschrift: Monopolkommission, Heilsbachstr. 16, D-53123 Bonn, Tel.: +49 (0)228/ 33-88-82-39, E-Mail: achim.buchwald@monopolkommission.bund.de

Der Autor dankt Benjamin Balsmeier, Alexander Dilger und zwei anonymen Gutachtern für wertvolle Anregungen und Kommentare. 